Sharif University of Technology
Scientia Iranica
SCIENTIA

Research Note

\title{
Integrated optimization of guidance and control parameters in a dual-spin flying vehicle
}

\author{
H. Nobahari* and M. Arab Kermani \\ Department of Aerospace Engineering, Sharif University of Technology, Zip Code 1458889694, Tehran, Iran.
}

Received 28 May 2015; received in revised form 8 May 2016; accepted 14 September 2016

\author{
KEYWORDS \\ Dual-spin flying \\ vehicle; \\ Dynamic modeling; \\ Integrated \\ optimization; \\ Proportional \\ navigation; \\ Tabu continuous ant \\ colony system.
}

\begin{abstract}
In this paper, integrated optimization of the guidance and control parameters of a dual-spin flying vehicle is presented. The vehicle is composed of two parts: a freerolling aft body, including the engine and the stabilizing fins, and a roll-isolated front body, including all necessary guidance and control equipment, such as onboard computer, control fins, and an inertial navigation system. After developing the governing equations of motion, control loops and the guidance algorithm are constructed. Controllers are designed for two operating points and the guidance algorithm consists of a midcourse and a terminal phase. In midcourse phase, a virtual target, located on the nominal trajectory, is followed using proportional navigation law; while, in the terminal phase, the vehicle is guided toward the real target. A new nonlinear saturation function is defined in order to saturate the maximum lateral acceleration command as a function of dynamic pressure. Finally, the integrated tuning of 23 guidance and control parameters is formulated as an optimization problem. The optimization problem is solved using a metaheuristic algorithm, called tabu continuous ant colony system. The performance of the optimized guidance and control system is evaluated using Monte Carlo simulations based on the complete nonlinear model.
\end{abstract}

(C) 2017 Sharif University of Technology. All rights reserved.

\section{Introduction}

Accuracy is one of the main challenges in design and manufacture of projectiles. Several factors deteriorate the accuracy of projectiles, the examples of which are thrust misalignment, inertial and geometrical asymmetries, atmospheric disturbances, etc. Axial rotation is one of the most common ways to reduce the errors caused by factors such as thrust misalignment and inertial asymmetries. However, the errors, caused by factors such as atmospheric turbulence and fuel mass

*. Corresponding author. Tel./Fax: +98 2166164636 E-mail addresses: nobahari@sharif.edu (H. Nobahari); mak51387@gmail.com (M.Arab Kermani)

doi: $10.24200 /$ sci. 2017.4306 tolerances, cannot be eliminated by axial rotation. Hence, final error of unguided flying vehicles remains significant and these errors increase as the range increases. In the past two decades, the use of low-cost guidance and control modules has been considered in such flying vehicles. However, these systems cannot work accurately when the vehicle has a high roll rate. One method to overcome this problem is to place a bearing mechanism between the nose and the main body. Then, while the aft body is rotating, rotation of the nose can be controlled by a canard set and, therefore, the guidance system can work properly if it is placed in the nose part.

To investigate and enhance the performance of dual-spin atmospheric projectiles, several works have been done [1-10]. In these works, several issues such as dynamic, stability, control, and guidance have been 
studied. Smith et al. [1] considered a spin-stabilized artillery projectile, modified to accommodate controllable canards, mounted on a non-rotating nose. They developed a dynamic model and employed this model to simulate the increase in accuracy. Both the forward and aft bodies were assumed to be mass balanced and a hydrodynamic bearing was used to couple the rolling motion of the bodies.

Costello and Peterson [2] reported a more general dynamic model for a finless dual-spin projectile considering inertially asymmetric forward and aft bodies. They considered a combination of hydrodynamic and roller bearing roll coupling between the forward and aft bodies. Then, by application of the linear theory for a rigid atmospheric projectile, the gyroscopic and dynamic stability factors were developed and compared with those of the single-body case. Also, the stability problem for a canard guided dual spin stabilized projectile was investigated by Wernert [3]. It was assumed that the projectile had a diagonal inertia matrix; the bearing applied only rolling moment to each body and the total center of mass was located on the longitudinal axis.

The work reported by Burchett et al. [4] modifies linear equations of [2] to predict swerving motion of a two-body flying vehicle in the presence of lateral pulse jets, exerted on front body. The swerving dynamic is solved in closed form, resulting in computationally simple algebraic expressions for the projectile trajectory under the influence of lateral pulse jets.

In references $[5,6]$, the dynamic modeling, control, and guidance of a two-body air to surface vehicle are reported. In these references, the same assumptions as those in [3] have been used to derive the equations of motion. The equations are linearized and, then, the roll and transversal autopilots are designed using the pole placement technique. In these references, the Proportional Navigation (PN) guidance, the linear homing guidance, and a parabolic homing guidance have been used.

Salman et al. [7] have used $H_{\infty}$ control to design the roll axis autopilot for the front body of a spinning flying vehicle. A coaxial motor has been used to position the nose at a desired angle with respect to the inertial frame.

Theodoulis et al. [8-10] have studied some guidance, control, and dynamic issues in a class of fin guided spin stabilized vehicles. In these works, the nose roll angle is controlled by a coaxial servomotor, whereas the lateral guidance and control are performed by two pairs of rotating canards placed on the nose $[9,10]$. The complete nonlinear equations of motion are stated in a non-rolling body frame [8-10]. Also, these works report equilibrium manifold computation [8,10], Linear Parameter Varying (LPV) modeling, and stability analysis [8-10]. In [9], a linear quadratic regulator has been designed and analyzed in order to construct the lateral autopilot. Gains have been scheduled and the effectiveness of the proposed algorithm has been investigated using nonlinear simulations. Also, in reference [10], local controllers are designed for several operating points of the flight envelope. The controllers are based on $H_{\infty}$ pole placement technique.

More recently, Yi Wang et al. [11,12] investigated a class of spin-stabilized projectiles with fixed canards. Formulae for the dynamic equilibrium angle and drift were derived. Then, the analytic solution to the deviation motion was obtained and it was shown that the control strategy could be developed through studying the influence of the dynamic equilibrium angle and the angle of attack. In [12], impact point deviation based on perturbation has been proposed. It has been shown that the proposed technique is suitable for the trajectory correction fuse.

Qing-Wei et al. [13] has proposed a guidance law for a class of dual-spin mortars that only use GPS receiver and geomagnetic measurements. Authors have divided trajectory to the ascending and descending segments and guidance law is designed for each segment separately.

In this paper, dynamic modeling, control, and guidance of a dual-spin canard guided flying vehicle are studied. A hydrodynamic bearing connects front and aft bodies. An Inertial Navigation System (INS), located in front body, is utilized for the positioning. The canard, embedded on the nose, is used to control roll angle of the nose part and to execute the lateral acceleration commands, issued by the guidance law. Dynamic model of the vehicle is derived in seven Degrees of Freedom (DoF). In all of the above works, the guidance and control modules are designed separately. To achieve more synergism between control and guidance loops, the parameters of the control and guidance loops are optimized in an integrated manner. For this purpose, the authors utilize a metaheuristic algorithm, called Tabu Continuous Ant Colony System (TCACS) [14], to simultaneously optimize the guidance and control parameters in order to minimize the final miss distance and the total control effort. The performance of the optimized guidance and control system is evaluated using Monte Carlo simulations based on the complete nonlinear model. For this purpose, errors in Specific Impulse $\left(I_{\mathrm{sp}}\right)$ and launch elevation angle, as well as the existence of cross wind during the initial parts of flight, are considered.

The rest of this paper is organized as follows: in Section 2, a brief description of the dynamic model is given. The structure of control loops is explained in Section 3. Guidance laws of the midcourse and terminal phases are introduced in Section 4. Section 5 is devoted to the definition of the optimization problem, the optimization algorithm, and the optimization 
results. Finally, concluding remarks are given in Section 6. A tensor derivation of the translational and rotational equations of motion is also made in Appendices A and B, respectively.

\section{Dynamic model of the dual-spin flying vehicle}

A general dynamic model for a dual-spin flying vehicle is developed in [2]. This reference uses a non-rotating body reference frame to develop the equations of motion. In the current study, since the front body, denoted by $B_{1}$, is controlled, a body reference frame, fixed to $B_{1}$, is taken to develop the equations of motion. It is assumed that the center of mass lies on the longitudinal symmetry axis of the vehicle and the inertia matrices of front and aft bodies are diagonal. The translational dynamic behavior of a dual-spin projectile is presented in $B_{1}$ as follows $[5,6]$ :

$$
\begin{aligned}
& \dot{u}=r v-q w+(1 / m)\left(X_{1}+X_{2}+X_{T_{2}}\right)-g \sin \theta, \\
& \dot{v}=p w-r u+(1 / m)\left(Y_{1}+Y_{2}^{\prime}+Y_{T_{2}}^{\prime}\right)+g \cos \theta \sin \phi, \\
& \dot{w}=q u-p v+(1 / m)\left(Z_{1}+Z_{2}^{\prime}+Z_{T_{2}}^{\prime}\right)+g \cos \theta \cos \phi,
\end{aligned}
$$

where $u, v$, and $w$ are components of the velocity vector; $p, q$, and $r$ are components of the angular velocity of $B_{1} ; \phi$ and $\theta$ are roll and pitch angles; $X_{1}, Y_{1}$, and $Z_{1}$ are components of the aerodynamic forces applied to the front body; $X_{2}, Y_{2}^{\prime}$, and $Z_{2}^{\prime}$ are components of the aerodynamic forces applied to the aft body; $X_{T_{2}}, Y_{T_{2}}^{\prime}$, and $Z_{T_{2}}^{\prime}$ are components of the thrust force applied to the aft body; $m$ is total mass of the flying vehicle; and $g$ is magnitude of the gravity vector. The derivation of Eqs. (1)-(3) is provided in Appendix A. Also, the following equations represent the angular dynamic behavior of a dual-spin projectile in $B_{1}$ :

$$
\begin{aligned}
\dot{p}= & \frac{1}{I_{x_{1}}}\left(L_{1}+b_{t} \dot{\phi}_{r}\right), \\
\dot{p}_{2}= & \frac{1}{I_{x_{2}}}\left(L_{2}+L_{T_{2}}-b_{t} \dot{\phi}_{r}\right), \\
\dot{q}= & p r(1-\mu)-p_{2} r \gamma+\frac{1}{I_{y}}\left(M_{1}+M_{2}^{\prime}-x_{1} Z_{1}\right. \\
& \left.+x_{2} Z_{2}^{\prime}+M_{T_{2}}^{\prime}+x_{2} Z_{T_{2}}^{\prime}\right), \\
\dot{r}= & -p q(1-\mu)+p_{2} q \gamma+\frac{1}{I_{y}}\left(N_{1}+N_{2}^{\prime}+x_{1} Y_{1}\right. \\
& \left.-x_{2} Y_{2}^{\prime}+N_{T_{2}}^{\prime}-x_{2} Y_{T_{2}}^{\prime}\right),
\end{aligned}
$$

where $I_{x_{1}}$ and $I_{x_{2}}$ are axial moments of inertia for front and aft bodies, respectively; $b_{t}$ is the viscous friction coefficient of the bearing; $\phi_{r}$ is the relative roll angle of aft body with respect to the front body; $p_{2}$ is the 1st component of angular velocity of aft body; $L_{1}, M_{1}$, and $N_{1}$ are components of the aerodynamic moments applied to front body; $L_{2}, M_{2}^{\prime}$, and $N_{2}^{\prime}$ are components of the aerodynamic moments applied to aft body; $L_{T_{2}}, M_{T_{2}}^{\prime}$, and $N_{T_{2}}^{\prime}$ are components of the thrust moment applied to aft body; and $x_{1}$ and $x_{2}$ are distances between the total center of mass and centers of masses of front and aft bodies, respectively. also $\mu$, $\gamma$, and $I_{y}$ are defined as follows:

$$
\begin{aligned}
& \mu=\frac{I_{x_{1}}}{I_{y}} \\
& \gamma=\frac{I_{x_{2}}}{I_{y}} \\
& I_{y}=I_{y_{1}}+I_{y_{2}}+\left(\frac{m_{1} m_{2}}{m}\right) d_{12}^{2},
\end{aligned}
$$

where $I_{y_{1}}$ and $I_{y_{2}}$ are transverse moments of inertia for front and aft bodies, respectively; $m_{1}$ and $m_{2}$ are masses of front and aft bodies, respectively; and $d_{12}$ is the distance between mass centers of front and aft bodies. Eqs. (4)-(7) are derived in Appendix B. Also, the aerodynamic forces and moments of the dual-spin vehicle are defined as:

$$
\begin{aligned}
& X=\bar{q} S C_{x}=X_{1}+X_{2}, \\
& Y=\bar{q} S C_{y}=Y_{1}+Y_{2}^{\prime}, \\
& Z=\bar{q} S C_{z}=Z_{1}+Z_{2}^{\prime}, \\
& L_{1}=\bar{q} S d C_{l_{1}}, \\
& L_{2}=\bar{q} S d C_{l_{2}}, \\
& M=\bar{q} S d C_{m}=M_{1}+M_{2}^{\prime}-x_{1} Z_{1}+x_{2} Z_{2}^{\prime}, \\
& N=\bar{q} S d C_{n}=N_{1}+N_{2}^{\prime}+x_{1} Y_{1}-x_{2} Y_{2}^{\prime},
\end{aligned}
$$

where $\bar{q}$ is the dynamic pressure, $S$ is the reference area, $d$ is the reference length, and $C_{i}\left(i=x, y, z, l_{1}, l_{2}, m, n\right)$ are the aerodynamic coefficients. In this paper, the aerodynamic coefficients are modeled as follows:

$$
\begin{aligned}
C_{x}= & C_{x_{0}}, \\
C_{y}= & C_{y_{\beta}} \beta+C_{y_{\delta_{r}}} \delta_{r}+C_{y_{r}} \frac{r d}{2 v_{M}}+C_{y_{\alpha}}(p) \alpha \\
& +C_{y_{p_{2}}} \frac{p_{2} d}{2 v_{M}}, \\
C_{z}= & C_{z_{\alpha}} \alpha+C_{z_{\delta_{e}}} \delta_{e}+C_{z_{q}} \frac{q d}{2 v_{M}}+C_{z_{\beta}}(p) \beta \\
& +C_{z_{p_{2}}} \frac{p_{2} d}{2 v_{M}},
\end{aligned}
$$




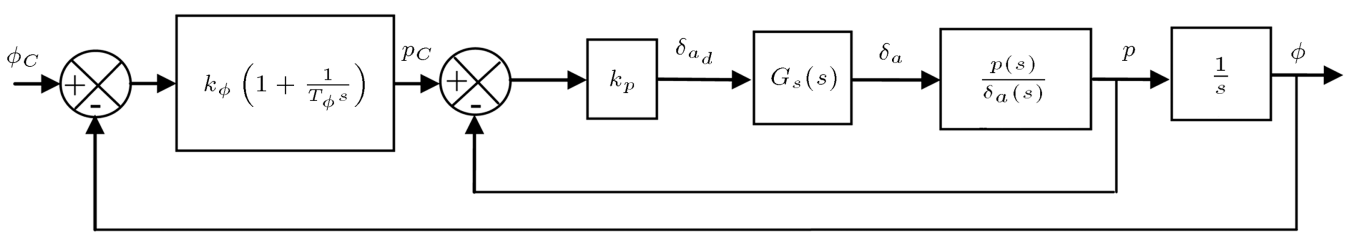

Figure 1. Roll control architecture.

$$
\begin{aligned}
C_{l_{1}}= & C_{l_{\delta_{a_{1}}}} \delta_{a_{1}}+C_{l_{p}} \frac{p d}{2 v_{M}}, \\
C_{l_{2}}= & C_{l_{\delta_{a_{2}}}} \delta_{a_{2}}+C_{l_{p_{2}}} \frac{p_{2} d}{2 v_{M}}, \\
C_{m}= & C_{m_{\alpha}} \alpha+C_{m_{\delta_{e}}} \delta_{e}+C_{m_{q}} \frac{q d}{2 v_{M}}+C_{m_{\beta}}(p) \beta \\
& +C_{m_{p_{2}}} \frac{p_{2} d}{2 v_{M}}, \\
C_{n}= & C_{n_{\beta} \beta} \beta+C_{n_{\delta_{r}}} \delta_{r}+C_{n_{r}} \frac{r d}{2 v_{M}}+C_{n_{\alpha}}(p) \alpha \\
& +C_{n_{p_{2}}} \frac{p_{2} d}{2 v_{M}},
\end{aligned}
$$

where $C_{i_{j}}\left(j=\alpha, \beta, \delta_{a_{1}}, \delta_{a_{2}}, \delta_{e}, \delta_{r}, p, p_{2}, q, r\right)$ are aerodynamic derivatives and $v_{M}$ is the magnitude of the velocity vector with respect to the Earth frame. Aft fins have a constant deflection angle of $\delta_{a_{2}}$ to apply the desired roll rate to aft body. Control fins are placed in the front body and their deflection angle $\left(\delta_{1}, \cdots, \delta_{4}\right)$ is related to $\delta_{a_{1}}, \delta_{e}$, and $\delta_{r}$ as [15]:

$$
\begin{gathered}
\left\{\begin{array}{l}
\delta_{e}=\frac{\delta_{2}-\delta_{4}}{2} \\
\delta_{r}=\frac{\delta_{1}-\delta_{3}}{2} \\
\delta_{a_{1}}=\frac{\delta_{1}+\delta_{2}+\delta_{3}+\delta_{4}}{4}
\end{array}\right. \\
\left\{\begin{array}{l}
\delta_{1}=\delta_{a_{1}}+\delta_{r} \\
\delta_{2}=\delta_{a_{1}}+\delta_{e} \\
\delta_{3}=\delta_{a_{1}}-\delta_{r} \\
\delta_{4}=\delta_{a_{1}}-\delta_{e}
\end{array}\right.
\end{gathered}
$$

In this work, the permitted range of deflection angles is defined as $\left|\delta_{i}\right|<10 \mathrm{deg}(i=1, \cdots, 4)$.

\section{Control loops}

In this section, the control loops of the front body are described. Since during the flight, the aerodynamic forces and moments vary with the change in dynamic pressure, $\bar{q}$, the controllers must be adapted, too. Therefore, the transfer functions are obtained at two operating points and the controllers are designed accordingly. The first set of controllers is used from the start time to time $t_{1}$ and from $t_{2}$ to the end of flight. The second set is used from time $t_{1}$ to $t_{2}$. The parameters $t_{1}$ and $t_{2}$ are optimized using the optimization algorithm. In the following, the structure of the control loops is introduced.

\subsection{Roll control}

Since the performance of INS, located in the front body, deteriorates in high angular rates, the roll motion of the front body must be controlled. Spin of the aft body can disturb the roll control of the front body. In this work, a roll control system is utilized to regulate the roll angle of the front body. For this purpose, a dual feedback control architecture is utilized, as shown in Figure 1. The roll rate is fed back from the INS gyro and roll position is calculated by INS. It is assumed that INS is ideal. The inner rate loop augments the aerodynamic damping, Zipfel [15], and the outer position loop executes the roll command. The inner loop uses a proportional controller with proportional gain, $k_{p}$, and the outer loop uses a proportional integral controller with a proportional gain, $k_{\phi}$, and an integral time constant, $T_{\phi}$. These parameters are obtained for each operating point using the optimization algorithm.

The servo transfer function is taken as:

$$
G_{s}(s)=\frac{\omega_{n}^{2}}{s^{2}+2 \xi_{s} \omega_{n} s+\omega_{n}^{2}},
$$

where $\omega_{n}$ and $\xi_{s}$ are natural frequency and damping ratio of servo, respectively. In this work, $\omega_{n}=200 \frac{\mathrm{rad}}{\mathrm{sec}}$ and $\xi_{s}$ is obtained in Section 5 using the optimization algorithm.

\subsection{Lateral acceleration control}

In order to convert the accelerations commands produced by the guidance law into physical motion of the control surfaces, an acceleration control system is needed. In this section, the acceleration control loop of pitch channel is described. Since the flying vehicle is axisymmetric, the yaw control system works in the same manner. Dual feedback control architecture is utilized, as shown in Figure 2. The pitch rate and acceleration are fed back from the INS gyro and the INS accelerometer, respectively. The inner rate loop augments the aerodynamic damping [15] and the outer acceleration loop executes the desired acceleration command. The inner loop uses a proportional controller with proportional gain, $k_{q}$, and the outer loop uses 


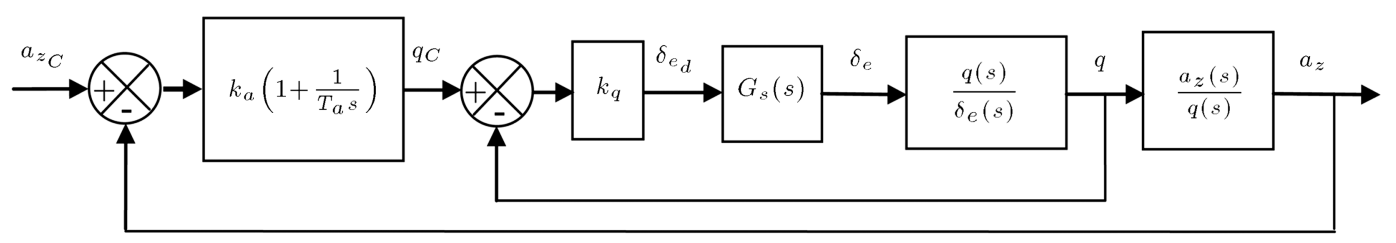

Figure 2. Lateral control architecture.

a proportional integral controller with a proportional gain, $k_{a}$, and an integral time constant, $T_{a}$. These parameters are obtained for each operating point using the optimization algorithm.

\section{Guidance algorithm}

In this section, details of the guidance algorithm are presented. The time sequence of the guidance algorithm is shown in Figure 3. The midcourse guidance algorithm is engaged at time, $t_{G}$, a little time after engine burnout, and gives the commands to the autopilot in order for the flying vehicle to follow a nominal ballistic trajectory. In this phase, the vehicle follows a virtual target using PPN. The virtual target is located on the nominal trajectory and has a time lead, $t_{L}$, with respect to the instantaneous position of the guided vehicle. The terminal guidance phase begins at time $t_{T G}$. In this phase, the vehicle is guided toward the real target using PPN. In Section 5, the parameters $t_{G}, t_{T G}$, and $t_{L}$ are optimized using the optimization algorithm.

In this study, gravity compensated PPN is used as guidance law. The acceleration commands in body coordinate system are calculated as follows:

$$
\mathbf{a}_{C}=N \boldsymbol{\omega}_{\perp} \times \mathbf{v}_{B}-\mathbf{g}
$$

where $N$ is the proportional navigation constant, $\mathbf{g}$ is the gravity vector, $\mathbf{v}_{B}$ is the velocity vector, and $\boldsymbol{\omega}_{\perp}$ is the vector of line of sight rate perpendicular to the line of sight defined as [15]:

$$
\boldsymbol{\omega}_{\perp}=\frac{\mathbf{s}_{B T} \times \mathbf{v}_{B T}}{\left\|\mathbf{s}_{B T}\right\|^{2}},
$$

where $\mathbf{s}_{B T}$ and $\mathbf{v}_{B T}$ are relative position and velocity of the flying vehicle with respect to the virtual target.

During the flight, the maximum lateral acceleration of the flying vehicle is a function of the dynamic pressure. Hence, the maximum lateral acceleration command, produced by the guidance law, is limited according to the current dynamic pressure. In this

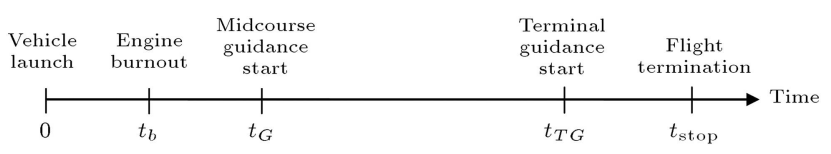

Figure 3. Time sequence of the guidance algorithm. paper, a nonlinear saturation function is defined as follows:

$$
a_{c, \text { max }}= \begin{cases}a_{\max } & \text { if } \bar{q} \geq \bar{q}_{\max } \\ \left(\frac{\bar{q}-\bar{q}_{\min }}{\bar{q}_{\max }-\bar{q}_{\min }}\right)^{p} a_{\max } & \text { if } \bar{q}_{\min } \leq \bar{q}<\bar{q}_{\max } \\ 0 & \text { if } \bar{q}<\bar{q}_{\min }\end{cases}
$$

where $\bar{q}_{\text {min }}$ is the minimum dynamic pressure over which the guidance algorithm issues commands, $\bar{q}_{\max }$ is the smallest dynamic pressure that can produce maximum lateral acceleration, $a_{\max }$ is the maximum lateral acceleration that can be applied during the flight, and $p$ is a constant. In the following section, the parameters $N, \bar{q}_{\max }, \bar{q}_{\min }, a_{\max }$, and $p$ are optimized using the optimization algorithm.

\section{Integrated optimization of the guidance and control parameters}

In this section, the integrated tuning of guidance and autopilot parameters is defined as an optimization problem. Then, a metaheuristic algorithm, called TCACS [14], is used to optimize the parameters. A nonlinear seven DoF flight simulation is used to calculate the cost function for each set of the guidance and control parameters generated by the optimization algorithm. Finally, a Monte Carlo simulation is performed to demonstrate the robustness of the proposed approach with respect to the gust and some other sources of error.

\subsection{Definition of the optimization problem}

In this section, the tuning of guidance and control parameters is defined as an optimization problem. The problem is to find the optimal values of $t_{1}, t_{2}, k_{p}$, $\cdots, T_{a}, \xi_{s}, t_{G}, t_{T G}, \cdots, a_{\max }$. Table 1 represents a complete list of these parameters with their search domains. The proportional navigation constant is usually selected between 2 and 4 [15]. The minimum and maximum limits of $\bar{q}_{\text {max }}$ and $\bar{q}_{\text {min }}$ are selected considering a typical dynamic pressure trend during the flight. Search domains of the controller gains and the time constants are determined based on a manual design of the control loops using root locus and bode diagram. Damping ratio of a second-order system is usually taken between 0.4 and 0.8 [16]. Finally, search domains of time parameters such as $t_{1}, t_{2}, t_{G}, t_{T G}$, and $t_{L}$ are set by considering the time of flight, burning time, etc. 
Table 1. Optimization parameters with their upper and lower bounds.

\begin{tabular}{cccccc}
\hline Parameter (unit) & Lower limit & Upper limit & Parameter (unit) & Lower Limit & Upper limit \\
\hline$a_{\max }\left(\frac{\mathrm{m}}{\mathrm{s}^{2}}\right)$ & 10 & 50 & $\frac{\bar{q}_{\min }}{\bar{q}_{\max }}$ & 0 & 0.1 \\
$k_{a_{2}}$ & -0.005 & 0 & $t_{G}(\mathrm{sec})$ & 4 & 10 \\
$\frac{k_{a_{1}}}{k_{a_{2}}}$ & 0.3 & 0.6 & $t_{T G}(\mathrm{sec})$ & 80 & 100 \\
$k_{p_{2}}$ & -0.5 & 0 & $t_{1}(\mathrm{sec})$ & 5 & 20 \\
$\frac{k_{p_{1}}}{k_{p_{2}}}$ & 0.1 & 0.2 & $t_{2}(\mathrm{sec})$ & 80 & 110 \\
$k_{q_{2}}$ & 0.5 & 1.5 & $T_{a_{2}}(\mathrm{sec})$ & 0 & 0.1 \\
$\frac{k_{q_{1}}}{k_{q_{2}}}$ & 0.2 & 0.5 & $T_{a_{1}}$ & 0.5 & 1 \\
$k_{\phi_{2}}$ & 5 & 15 & $T_{\phi_{2}}(\mathrm{sec})$ & 0 & 0.5 \\
$k_{\phi_{1}}$ & 0.5 & 1 & $T_{\phi_{1}}$ & 0 & 1 \\
$\bar{k}_{\phi_{2}}$ & 0 & $\xi_{s}$ & 0.4 & 0.8 \\
$p$ & 2 & 4 & $t_{L}(\mathrm{sec})$ & 0 & 10 \\
$\bar{q}_{\max }(\mathrm{Pa})$ & 500000 & 600000 & & & \\
\hline
\end{tabular}

In this work, miss distance and control effort are minimized to optimize the integrated performance of the guidance and control systems. Moreover, to obtain an acceptable roll control for the front body, the integral of absolute roll angle of the front body is added to the cost function as a penalty and the cost function is defined as follows:

$$
\text { Cost }=20^{*} \text { Miss }+ \text { Effort }+0.01^{*} \int\left|\phi_{1}\right| d t,
$$

where Miss is the final miss distance and Effort is the control effort defined as:

$$
\text { Effort }=\sqrt{a_{y_{C}}^{2}+a_{z_{C}}^{2}} .
$$

This paper engages application of TCACS [14] to optimize the guidance and control parameters in order to minimize the cost function. Figure 4 shows the iterative optimization process. TCACS continually guesses the optimal values of the parameters. After generation of any new guess, a complete nonlinear seven DoF simulation of the flying vehicle is performed and the cost function is calculated. The measured cost

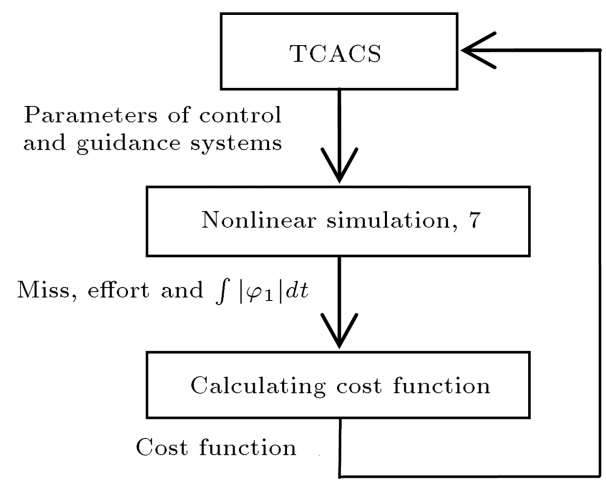

Figure 4. Optimization procedure using TCACS algorithm . is fed back to TCACS to help it converge on the optimal set of parameters.

\subsection{TCACS algorithm}

TCACS is a hybrid optimization algorithm for minimization of continuous multi-minima functions [14]. It is a combination of the well-known Ant Colony Optimization (ACO) [17], and Tabu Search (TS) [18], approaches. TCACS incorporates the concepts of promising list, tabu list, and tabu balls from TS into the framework of Continuous Ant Colony System (CACS) [19].

In a continuous optimization problem, it is desired to find the global minimum of a function, $f$, within a given interval $[a, b]$, in which the minimum occurs at a point $x_{s}$. In general, $f$ can be a multivariable function defined on a subset $R^{n}$ delimited by $n$ intervals $\left[a_{i}, b_{i}\right]$, $i=1, \cdots, n$.

Figure 5 shows the general iterative structure of TCACS. A continuous pheromone model is used to gradually guide the ants toward the global minimum point. This pheromone model is in fact a strategy

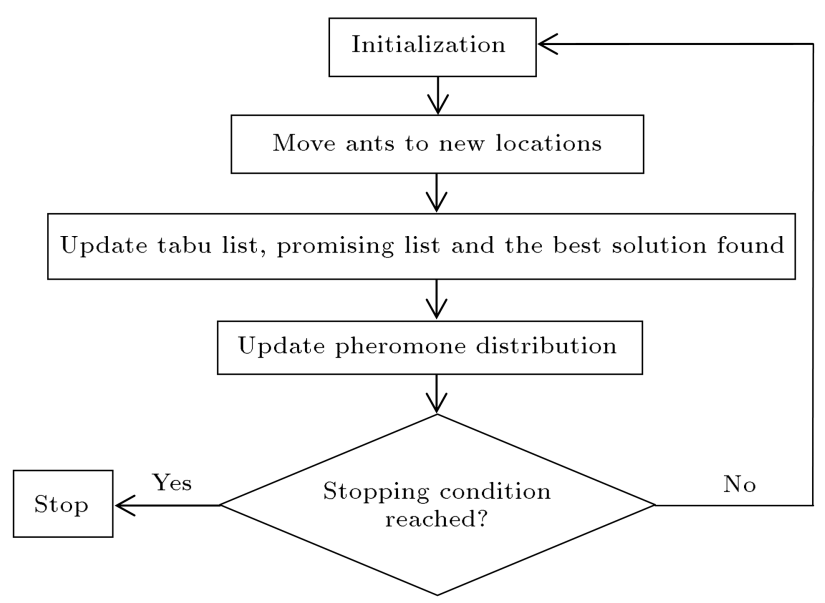

Figure 5. Flowchart of TCACS algorithm. 
Table 2. Optimal values of the guidance and control parameters.

\begin{tabular}{cccccc}
\hline Parameter (unit) & Value & Parameter (unit) & Value & Parameter (unit) & Value \\
\hline$a_{\max }\left(\frac{\mathrm{m}}{\mathrm{s}^{2}}\right)$ & 19.56 & $\frac{k_{\phi_{1}}}{k_{\phi_{2}}}$ & 0.726 & $t_{2}(\mathrm{sec})$ & 97.02 \\
$k_{a_{2}}$ & -0.0012 & $p$ & 0.57 & $T_{a_{2}}(\mathrm{sec})$ & 0.0097 \\
$\frac{k_{a_{1}}}{k_{a_{2}}}$ & 0.397 & $N$ & 2.04 & $\frac{T_{a_{1}}}{T_{a_{2}}}$ & 0.616 \\
$k_{p_{2}}$ & -0.158 & $\bar{q}_{\max }(\mathrm{Pa})$ & 571073.2 & $T_{\phi_{2}}(\mathrm{sec})$ & 0.468 \\
$\frac{k_{p_{1}}}{k_{p_{2}}}$ & 0.148 & $\frac{\bar{q}_{\min }}{\bar{q}_{\max }}$ & 0.0828 & $\frac{T_{\phi_{1}}}{T_{\phi_{2}}}$ & 0.77 \\
$k_{q_{2}}$ & 0.78 & $t_{G}(\mathrm{sec})$ & 5.01 & $\xi_{s}$ & 0.626 \\
$\frac{k_{q_{1}}}{k_{q_{2}}}$ & 0.43 & $t_{T G}(\mathrm{sec})$ & 95.35 & $t_{L}(\mathrm{sec})$ & 3.66 \\
$k_{\phi_{2}}$ & 7.33 & $t_{1}(\mathrm{sec})$ & 16.21 & & \\
\hline
\end{tabular}

to assign a continuous probability distribution to the whole search space and to update it as the algorithm progresses. During any iteration, ants move from their current positions to the new destinations according to the current pheromone distribution. The destinations are chosen using a random generator with normal probability distribution function. The values of the objective function are calculated in these new points and some knowledge about the problem is acquired, which is used to update the pheromone distribution.

For each dimension of the search space, a normal probability distribution function is used to model the pheromone aggregation around the current best point. Therefore, for $\mathbf{x}=\left(x_{1}, x_{2}, \cdots, x_{k}\right)$ being an arbitrary point within the solution space, and $\mathbf{x}^{*}=\left(x_{1}^{*}, x_{2}^{*}, \cdots, x_{k}^{*}\right)$ being the current best point, the pheromone intensities are given by $n$ normal distribution functions in the following form:

$$
\tau\left(x_{i}\right)=e^{-\frac{\left(x_{i}-x_{i}^{*}\right)^{2}}{2 \sigma_{i}^{2}}}
$$

where $\sigma_{i}$ is the variance of the normal distribution corresponding to the $i$ th dimension of the search space. The algorithm updates $x_{i}^{*}$ and $\sigma_{i}$ as it proceeds and the pheromone distribution over the search space gradually changes.

TCACS uses the concept of tabu and promising lists. Simply, a specified number of the best points, found from the beginning of the algorithm up to the current iteration, form the promising list. Likewise, a specified number of the worst points, found so far, constitute the tabu list. Each member of the tabu list is the center of a tabu ball, the size of which is updated during iterations. Tabu balls specify circular, spherical, and, in general, hyper spherical regions within the search space. Ants are not allowed to select any point inside the tabu balls while they are choosing their new destinations.

\subsection{Optimization results}

After 200 iterations of the optimization process, the optimal values of guidance and control parameters are
Table 3. Initial conditions of the nominal trajectory.

\begin{tabular}{lc}
\hline \multicolumn{1}{c}{ Parameter (unit) } & Value \\
\hline Altitude $(\mathrm{m})$ & 0 \\
Latitude (deg) & 34 \\
Launch azimuth angle (deg) & 0 \\
Launch elevation angle (deg) & $56.6+1$ \\
Longitude (deg) & 54 \\
\hline
\end{tabular}

obtained as represented in Table 2. The value of cost function corresponding to these optimal values is 173.07 .

In order to evaluate the performance of the optimized guidance and control system, a nonlinear seven DoF numerical simulation is performed. Table 3 represents the initial conditions by which the nominal trajectory of unguided vehicle is generated. The launch elevation angle of the guided vehicle is taken to be one degree higher than that of the nominal trajectory.

Figures 6 to 15 demonstrate the simulation results. Figure 6 shows the trajectory of flying vehicle in vertical and horizontal planes. Figure 7 shows the

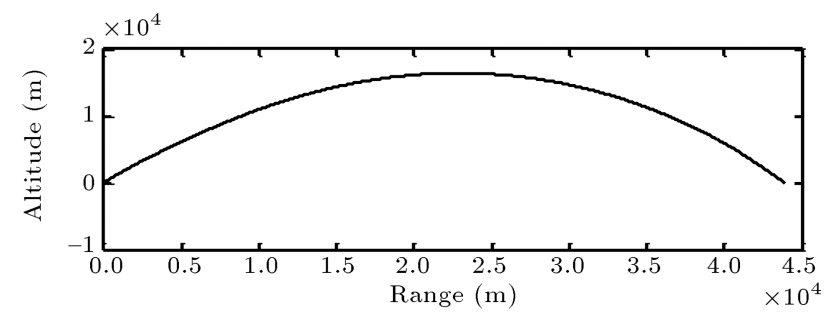

(a)

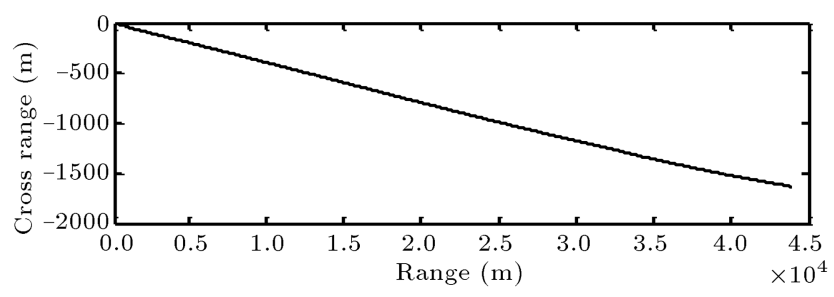

(b)

Figure 6. Flight trajectory: (a) Vertical plane and (b) horizontal plane. 


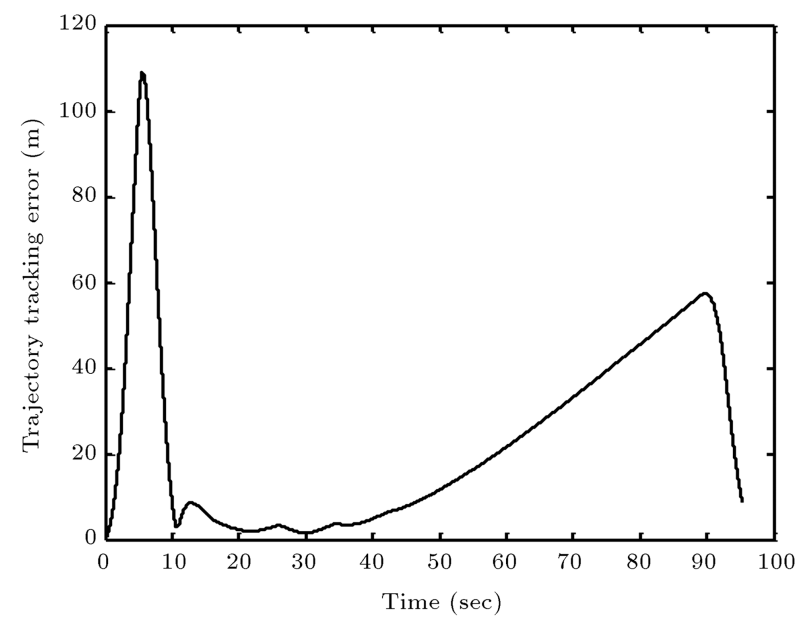

Figure 7. Distance between the real and nominal trajectories.

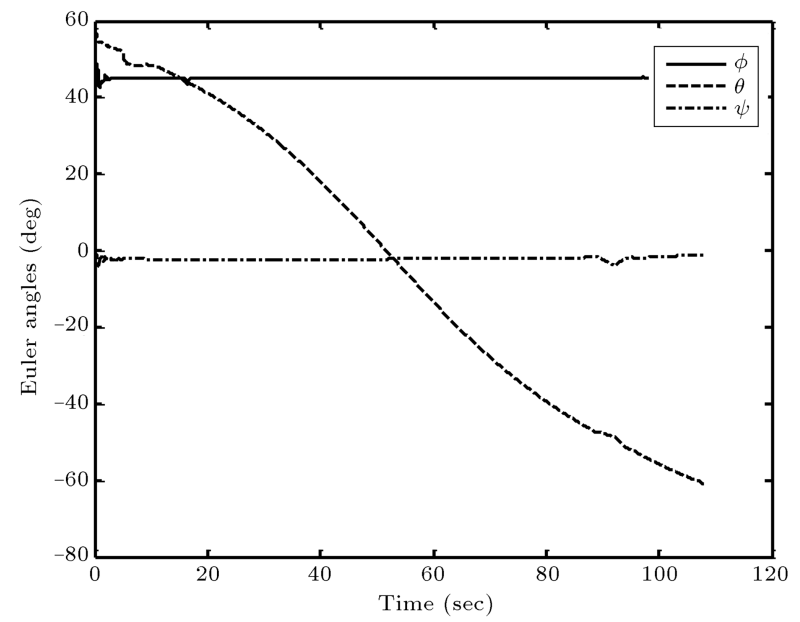

Figure 8. Euler angles of the front body versus time.

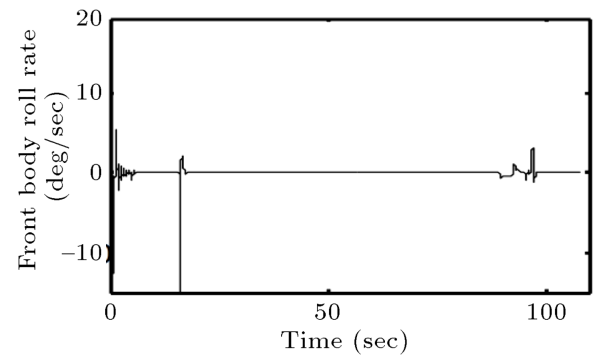

(a)

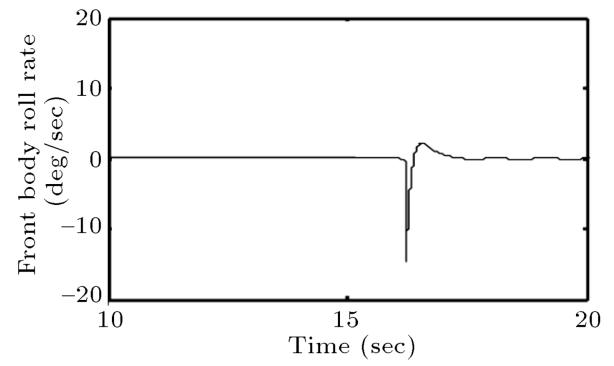

(c)

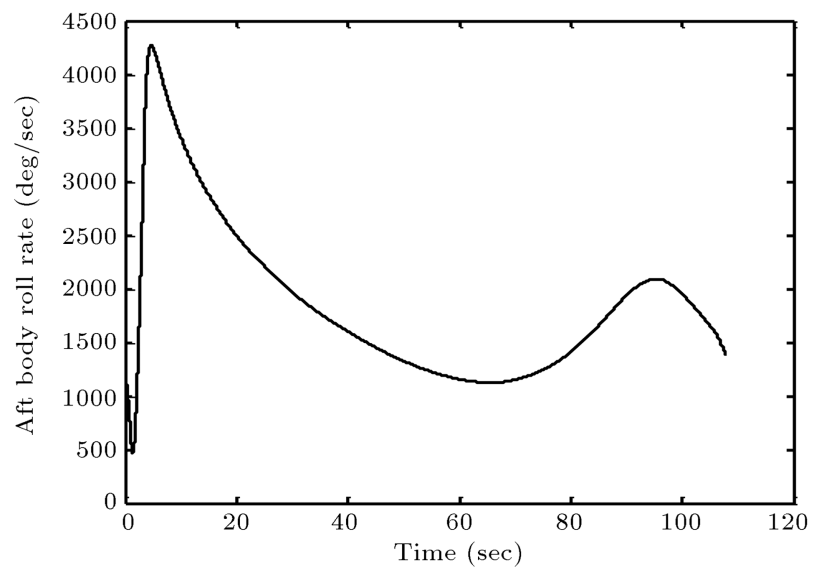

Figure 10. Roll rate of the aft body versus time.

instantaneous error between the nominal and guided trajectories. It shows that the guided vehicle has a little error in following the nominal trajectory. This error first grows since there is no guidance in the first seconds of flight. After the midcourse guidance algorithm is engaged at time $t_{G}=5.01 \mathrm{sec}$, the trajectory tracking error is controlled. This error increases with increasing the altitude, which reduces the control power. But, by approaching the surface of Earth, it is reduced again. Finally, when the terminal guidance of the flying vehicle is started at time $t_{T G}=95.35 \mathrm{sec}$, trajectory tracking is stopped.

Figure 8 shows the time history of Euler angles of the front body. The good regulation of the roll angle with zero steady state error with respect to the initial $\phi_{0}=45 \mathrm{deg}$ can be found in this figure. Figure 9 shows roll rate of the front body in different time slots. It again shows the good performance of the roll

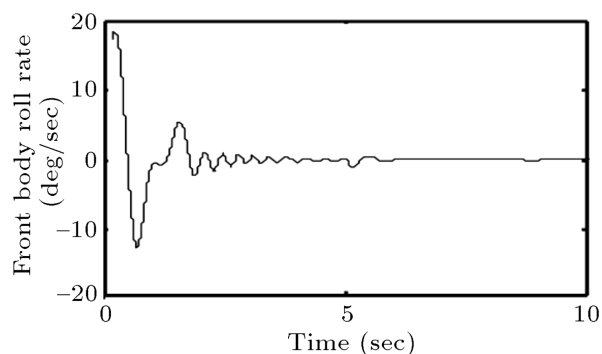

(b)

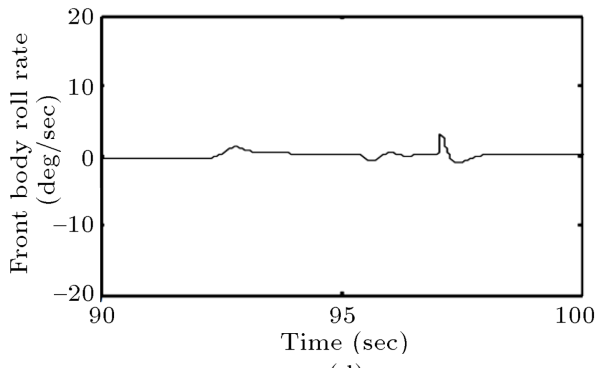

(d)

Figure 9. Roll rate of the front body versus time: (a) All times, (b) from $t=0$ to $t=10 \mathrm{sec}$, (c) from $t=10$ to $t=20$ sec and (d) from $t=90$ to $t=100$. 


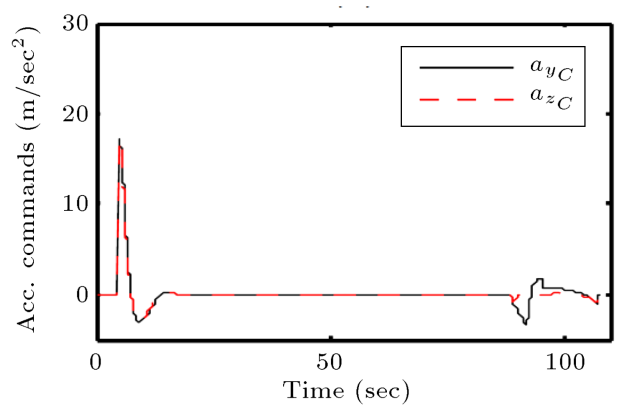

(a)

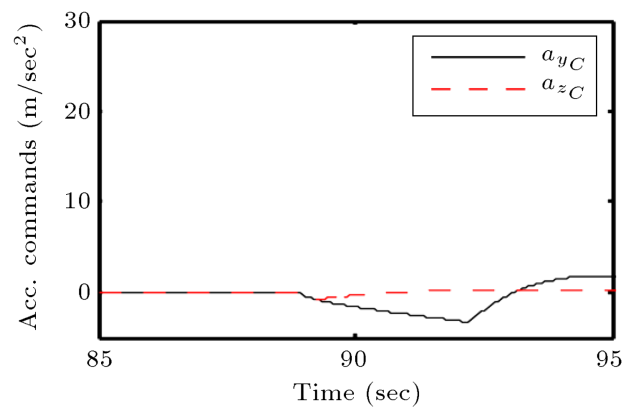

(c)

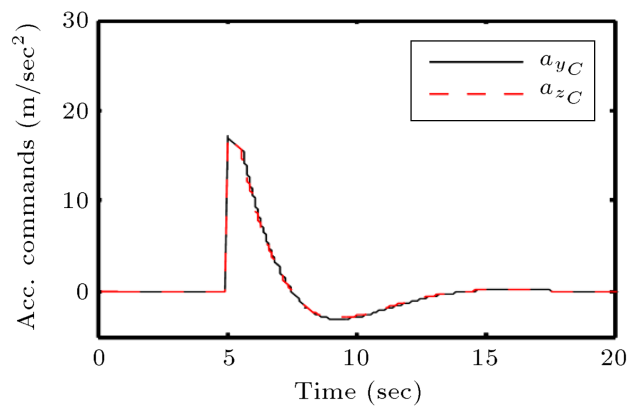

(b)

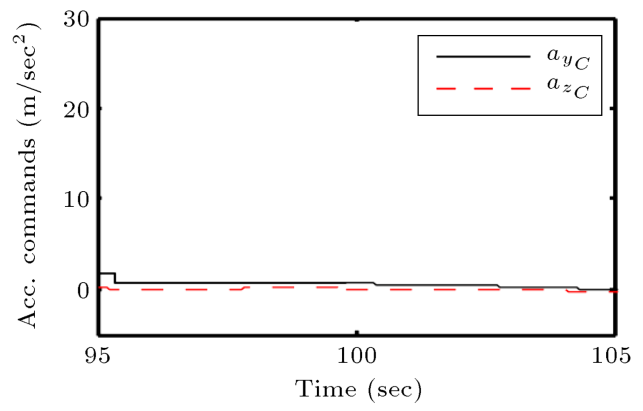

(d)

Figure 11. Acceleration commands versus time: (a) All times, (b) from $t=0$ to $t=20$ sec, (c) from $t=85$ to $t=95$ sec, and (d) from $t=95$ to $t=105 \mathrm{sec}$.

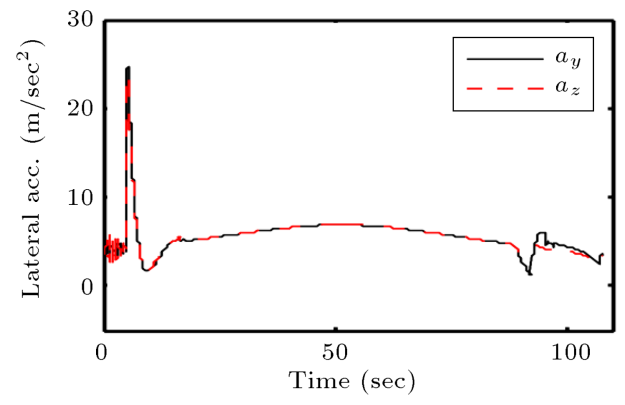

(a)

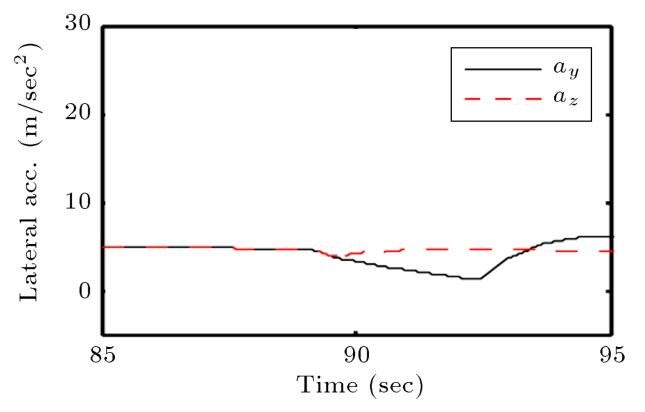

(c)

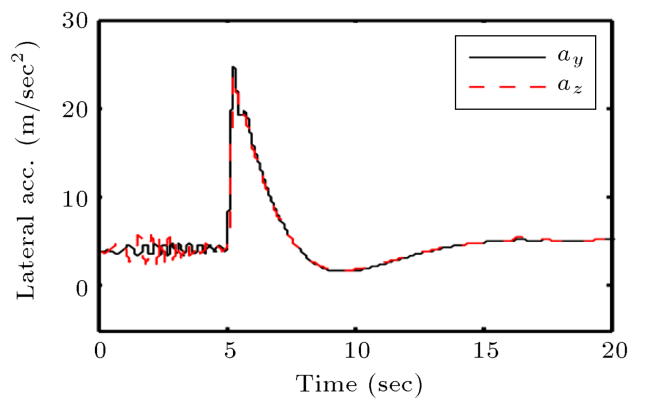

(b)

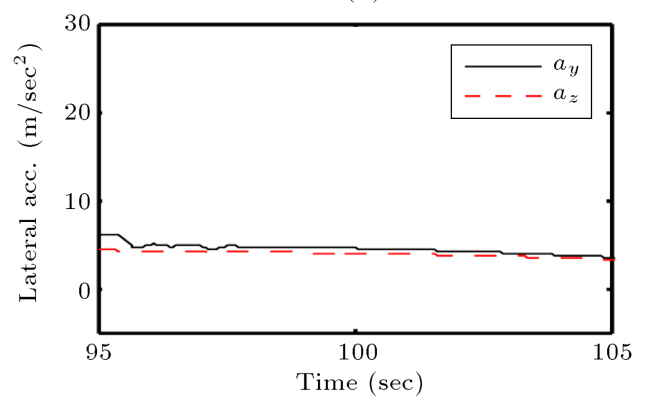

(d)

Figure 12. Lateral accelerations versus time: (a) All times, (b) from $t=0$ to $t=20 \mathrm{sec}$, (c) from $t=85$ to $t=95$ sec, and (d) from $t=95$ to $t=105 \mathrm{sec}$.

controller. Figure 10 shows the roll rate of the aft body. It should be noted that the aft body freely rolls due to the incidence angle of the aft fins. Therefore, the trend of roll rate is similar to that of the vehicle velocity. It increases up to the engine burnout. Then, it decreases up to the trajectory peak. In the second part of the trajectory, the velocity first increases due to the gravitational acceleration and, finally, decreases due to the increase in air density.

Figure 11 shows the acceleration commands produced by the guidance law and Figure 12 represents the total aerodynamic and gravity accelerations exerted on the front body. Comparing Figures 11 and 12 shows that the tracking of the acceleration commands 


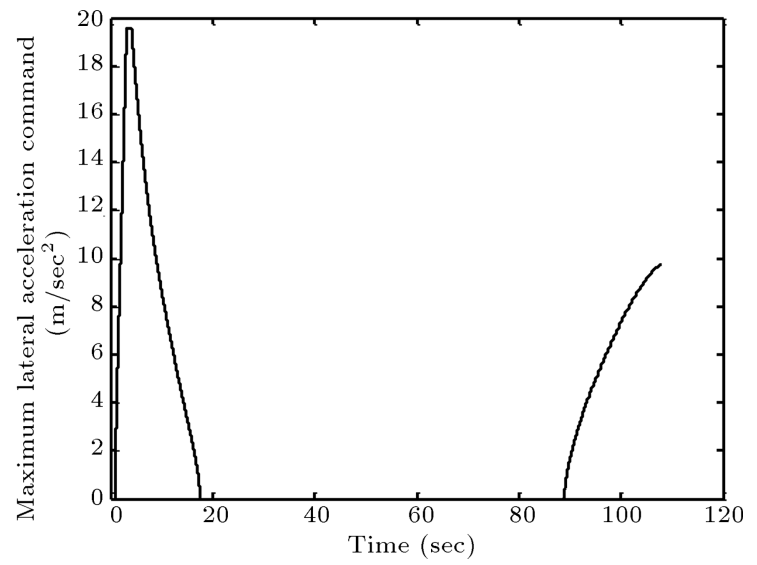

Figure 13. Maximum lateral acceleration command versus time.

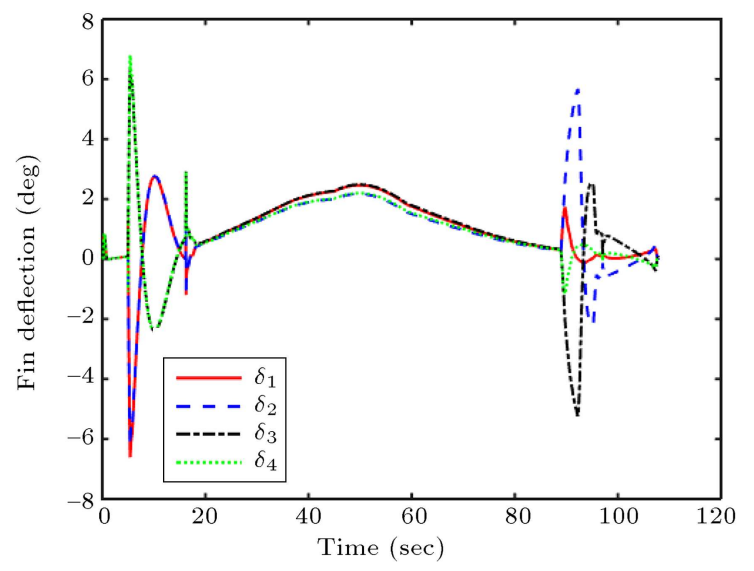

Figure 14. Fins deflection angle versus time.

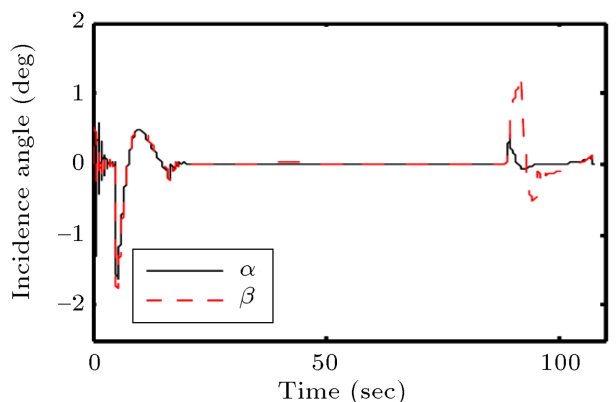

(a)

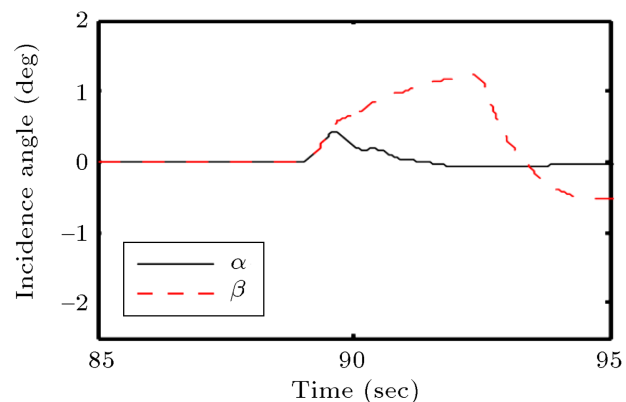

(c) is satisfactory. At the beginning of the guidance phase, the acceleration commands are about $1.8 \mathrm{~g}$. Then, increasing the altitude reduces the control power and, consequently, the acceleration commands. Figure 13 shows the permitted maximum acceleration command, calculated using Eq. (30). In the terminal phase, the acceleration commands are less than $0.5 \mathrm{~g}$ in order to hit the real target. Figure 14 represents the time history of fins deflection angle and shows that deflection angles are within the permitted range of $[-10,10] \mathrm{deg}$. Finally, incidence angles of the front body are shown in Figure 15. In this simulation, the final miss distance and control effort are $0.49 \mathrm{~m}$ and $93.36 \mathrm{~m} / \mathrm{s}$.

\subsection{Error analysis}

In order to investigate the robustness of the proposed method in the presence of error sources, a Monte Carlo simulation is performed. The simulation could be utilized for the flying vehicle and the environmental uncertainties. In this paper, errors in specific impulse and launch elevation angle are considered as the flying vehicle uncertainties and the cross wind is considered as an environmental uncertainty. These errors are generated by random numbers with Gaussian distribution. These random numbers and how they are applied to the simulation are shown in Table 4. The lateral wind is applied from the start time of simulation to $t=10 \mathrm{sec}$.

Figures 16-18 respectively show the Circular Error Probability (CEP) of the unguided and guided flying vehicle for 100 iterations the of Monte Carlo simulation. The radius of circle, centered about the nominal target,

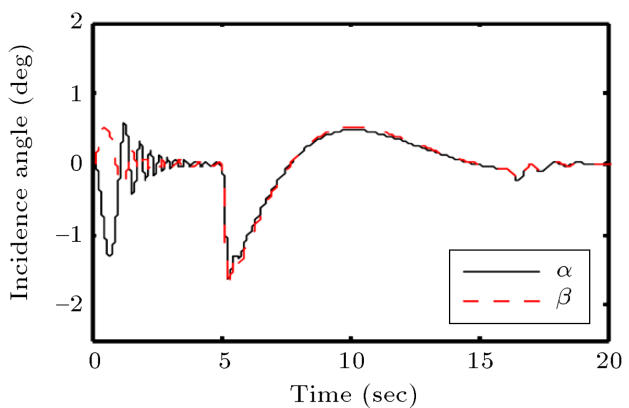

(b)

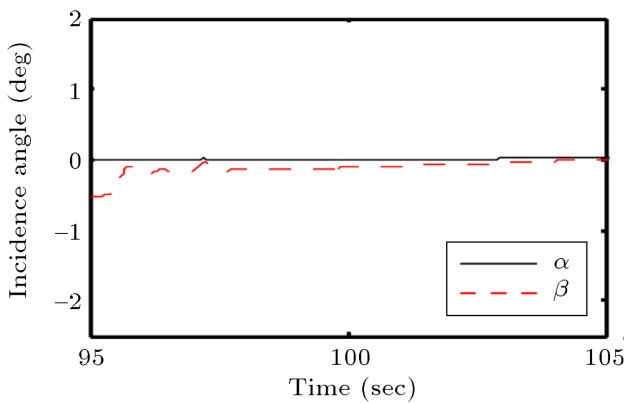

(d)

Figure 15. Incidence angles versus time: (a) All times, (b) from $t=0$ to $t=20 \mathrm{sec}$, (c) from $t=85$ to $t=95 \mathrm{sec}$, and (d) from $t=95$ to $t=105$ sec. 
Table 4. Definition of the random numbers and the way they are applied.

\begin{tabular}{lcccl}
\hline & Mean & Standard deviation & Unit & \multicolumn{1}{c}{ Application } \\
\hline Random number 1 & 1 & 0.01 & - & Multiplied by the specific impulse \\
Random number 2 & 0 & 1 & $\mathrm{deg}$ & Added to the elevation launch angle \\
Random number 3 & 0 & 2 & $\mathrm{~m} / \mathrm{s}$ & Considered as a lateral wind \\
\hline
\end{tabular}

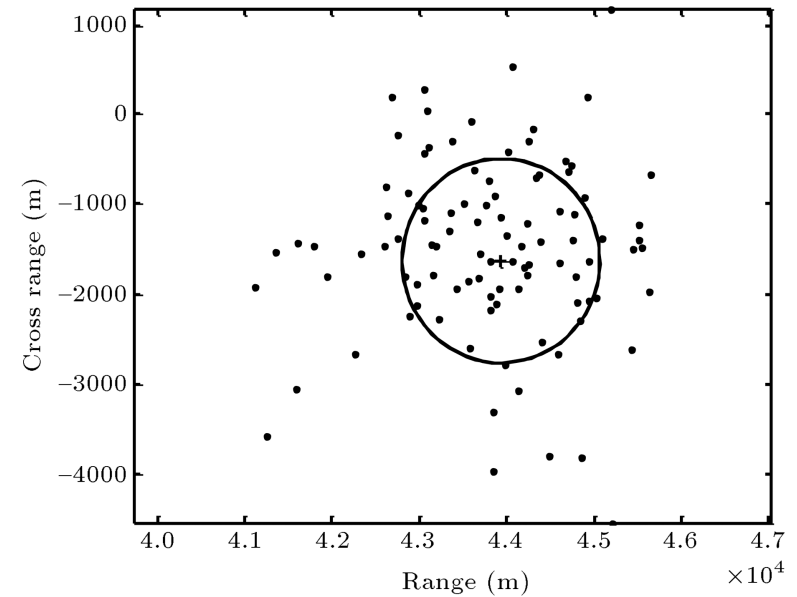

Figure 16. CEP of unguided flying vehicle.

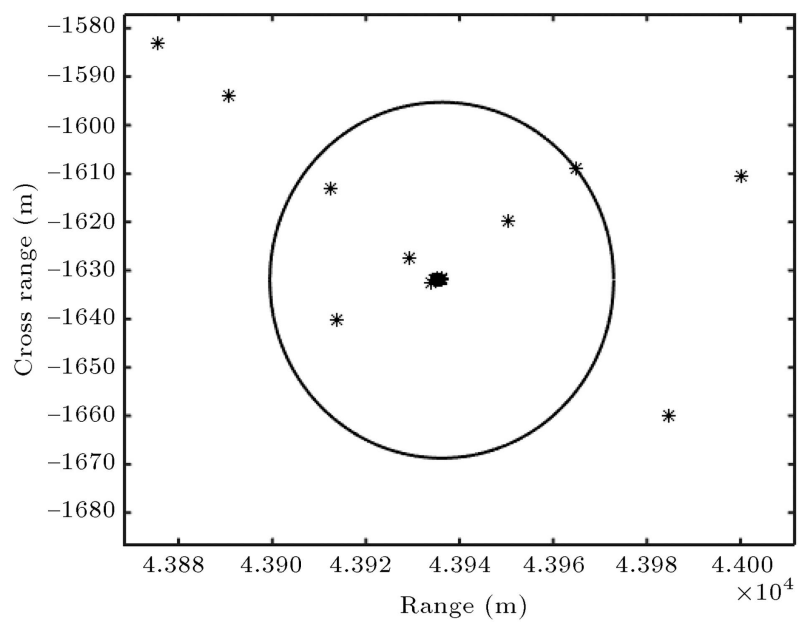

Figure 17. CEP of guided vehicle for the non-optimal design obtained after 50 evaluations.

is $1131 \mathrm{~m}$ for the unguided vehicle and $36.72 \mathrm{~m}$ for the non-optimal design, obtained after 50 evaluations of the cost function by the optimization algorithm; also, it is $0.58 \mathrm{~m}$ for the optimized guided vehicle. It should be noted that the authors have avoided taking INS errors into account in order to be able to investigate the pure performance of the guidance and control algorithms. Therefore, in a real application, the INS errors will increase the CEP of guided vehicle.

\section{Conclusion}

In this study, an integrated optimal tuning of the guidance and control parameters of a dual-spin fly-

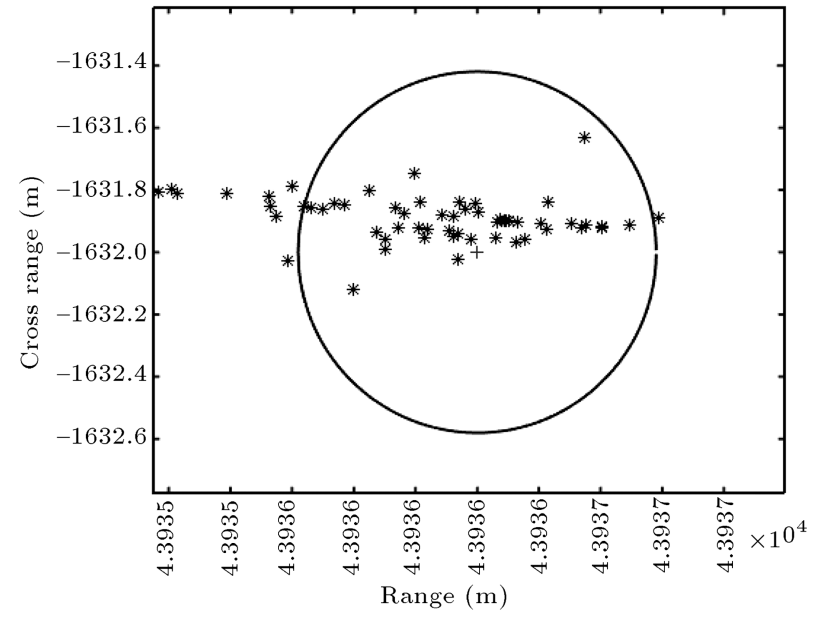

Figure 18. CEP of guided vehicle for the optimal design obtained after 200 evaluations.

ing vehicle using an effective evolutionary algorithm was investigated. The complete seven DoF nonlinear equations of motion were derived in tensor form (Appendices $\mathrm{A}$ and $\mathrm{B}$ ). The structure of the control loops, utilized for the front body, was given. Since the aerodynamic forces and moments changed as a function of the dynamic pressure, the controllers were parameterized in two operating points. The guidance algorithm was designed in midcourse and terminal phases. A trajectory tracking guidance algorithm, based on PN was utilized in the midcourse phase. A new nonlinear saturation function was defined in order to saturate the maximum lateral acceleration commands as a function of the dynamic pressure. Afterwards, integrated tuning of the guidance and control parameters was defined as an optimization problem. Then, using TCACS, the optimal values of these parameters were obtained. Simulation results showed good performance of the optimized guidance and control system both in trajectory tracking in midcourse phase and in the terminal intercept. Finally, Monte Carlo simulations were performed in the presence of a lateral wind as an environmental uncertainty and other error sources such as errors in specific impulse and launch elevation angle. The results of Monte Carlo simulations showed robustness of the optimized guidance and control system.

\section{Nomenclature}

$\mathbf{a}_{C}$

Vector of the acceleration commands in body coordinate system 


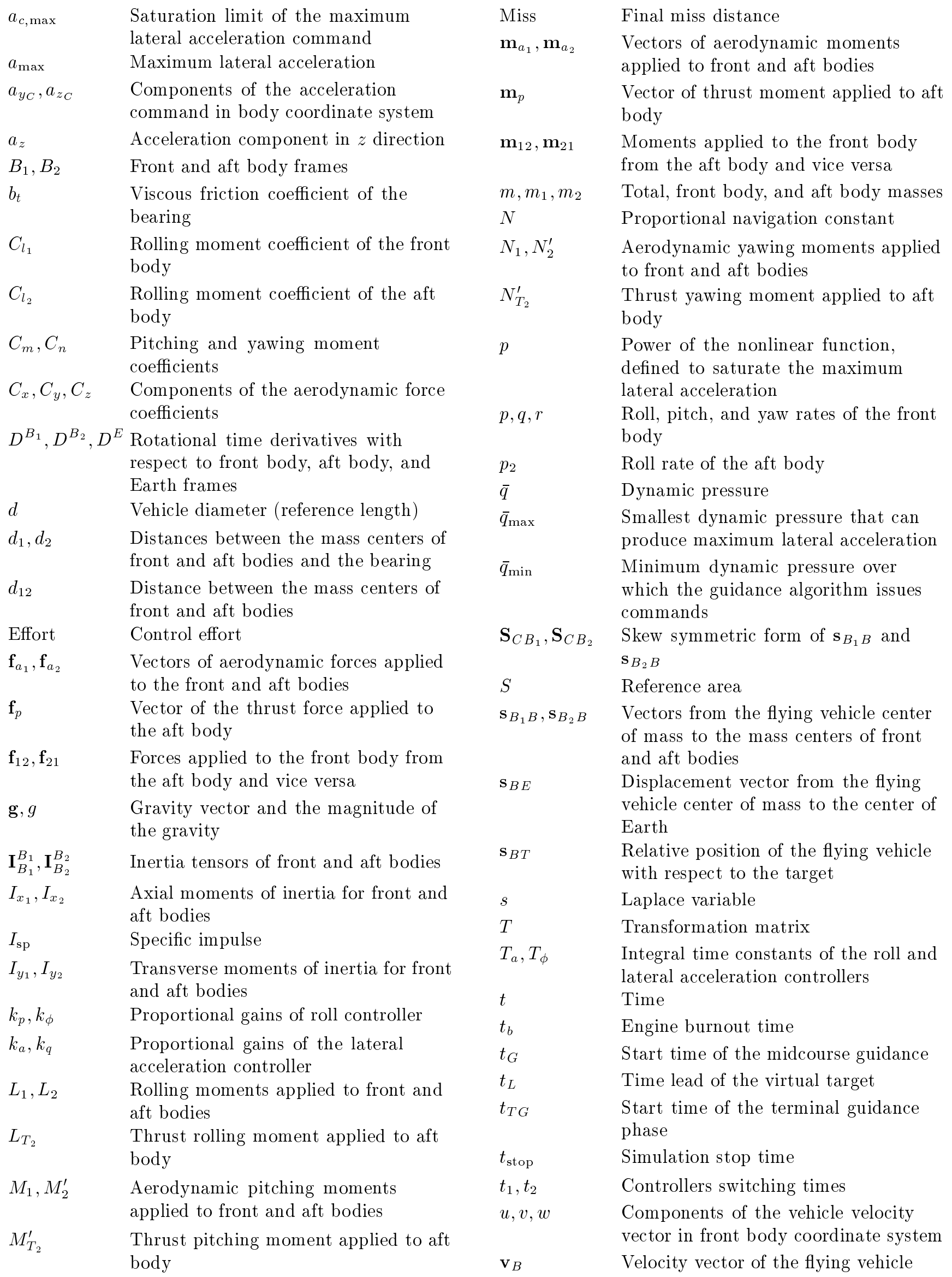


$\mathbf{v}_{B_{1}}^{B_{1}}, \mathbf{v}_{B_{2}}^{B_{1}} \quad$ Velocity vectors of the front and aft bodies with respect to the front body frame

$\mathbf{v}_{B}^{E} \quad$ The vehicle velocity vector with respect to Earth frame

$\mathbf{v}_{B_{1}}^{E}, \mathbf{v}_{B_{2}}^{E} \quad$ Velocity vectors of the front and aft bodies with respect to Earth frame

$v_{M} \quad$ Magnitude of the vehicle velocity vector with respect to Earth frame

$X_{1}, X_{2} \quad$ The first components of aerodynamic forces applied to front and aft bodies

$X_{T_{2}} \quad$ The first component of thrust force applied to aft body

$x_{1}, x_{2} \quad$ Distances between the total center of mass and the mass centers of front and aft bodies

$Y_{1}, Y_{2}^{\prime} \quad$ The second components of aerodynamic forces applied to front and aft bodies

$Y_{T_{2}}^{\prime} \quad$ The second component of thrust force applied to aft body

$Z_{1}, Z_{2}^{\prime} \quad$ The third components of aerodynamic forces applied to front and aft bodies

$Z_{T_{2}}^{\prime} \quad$ The third component of thrust force applied to aft body

$\alpha, \beta \quad$ Angle of attack and side slip angle

$\delta_{a_{1}}, \delta_{e}, \delta_{r} \quad$ Control deflections of front fins

$\delta_{a_{2}}$

$\phi, \theta, \psi$

$\xi_{s}$

$\sigma$

$\tau$

$\phi_{r}$

Fixed deflection of aft fins

Euler angles

Damping ratio of servo

Variance of normal distribution

Pheromone distribution function

Relative roll angle of aft body with respect to front body

$\boldsymbol{\Omega}^{B_{1} E}, \boldsymbol{\Omega}^{B_{2} E} \quad$ Skew symmetric forms of $\boldsymbol{\omega}^{B_{1} E}$ and $\boldsymbol{\omega}^{B_{2} E}$

$\boldsymbol{\omega}^{B_{2} B_{1}} \quad$ Angular velocity of aft body with respect to front body

$\boldsymbol{\omega}^{B_{1} E}, \boldsymbol{\omega}^{B_{2} E} \quad$ Angular velocities of front and aft bodies with respect to Earth frame

$\omega_{n} \quad$ Natural frequency of servo

\section{References}

1. Smith, J.A., Smith, K.A. and Topliffe, R. "Feasibility study for application of modular guidance and control units to existing ICM projectiles", Final Technical Report, U.S. Army Armament Research and Development Command, USA (1978).

2. Costello, M. and Peterson, A. "Linear theory of a dual-spin projectile in atmospheric flight", Journal of Guidance, Control, and Dynamics, 23, pp. 789-797 (2000).
3. Wernert, P. "Stability analysis for canard guided dualspin stabilized projectiles", In AIAA Atmospheric Flight Mechanics Conference, Chicago, USA, pp. 1-24 (2009).

4. Burchett, B., Peterson, A. and Costello, M. "Prediction of swerving motion of a dual-spin projectile with lateral pulse jets in atmospheric flight", J. Mathematical and Computer Modeling, 35, pp. 821-834 (2002).

5. Özkan, B. "Dynamic modeling, guidance and control of homing missiles", PhD Thesis, Middle East Technical University, Turkey (2005).

6. Özkan, B. and Özgören, M.K. "Implementation of linear homing guidance law on a two-part homing missile", In Proceedings of the 17th World Congress the International Federation of Automatic Control, Seoul, Korea, pp. 13999-14004 (2008).

7. Salman, M., Chang, B. and Ilg, M. "Control of spinning flying vehicles utilizing partial body despin techniques", In IEEE rth Asian Control Conference, Honk Kong, China, pp. 326-331 (2009).

8. Wernert, P. and Theodoulis, S. "Modeling and stability analysis for a class of $155 \mathrm{~mm}$ spin-stabilized projectiles with Course Correction Fuse (CCF)", In AIAA Atmospheric Flight Mechanics Conference, Portland, OR, pp. 1-14 (2011).

9. Theodoulis, S. and Wernert, P. "Flight control for a class of $155 \mathrm{~mm}$ spin-stabilized projectiles with Course Correction Fuse (CCF)", In AIAA Guidance, Navigation and Control Conference and Exhibit, Portland, OR, pp. 1-10 (2011).

10. Theodoulis, S., Gassmann, V., Wernert, P., Dritsas, L., Kitsios, I. and Tzes, A. "Guidance and control design for a class of spin-stabilized fin-controlled projectiles", Journal of Guidance, Control, and Dynamic, 36, pp. 517-531 (2013).

11. Yi, W., Wei-dong, S., Qing-wei, G., Xie-en, S. and Gaang, W. "Correction mechanism analysis for a class of spin-stabilized projectiles with fixed canards", Engineering Letters, 23(4), EL_23_4_05, pp. 1-8 (2015).

12. Yi, W., Wei-dong, S., Dan, F. and Qing-wei, G. "Guidance and control design for a class of spinstabilized projectiles with a two-dimensional trajectory correction fuze", International Journal of Aerospace Engineering, 2015, pp. 1-15 (2015).

13. Qing-wei, G., Wei-dong, S., Yi, W. and Zhi-cai, L. "Guidance law design for a class of dual-spin mortars", International Journal of Aerospace Engineering, 2015, pp. 1-12 (2015).

14. Karimi, A., Nobahari, H. and Sairry, P. "Continuous ant colony system and tabu search algorithms hybridized for global minimization of continuous multiminima functions", Computational Optimization and Applications (COAP) J., 45, pp. 639-661 (2010).

15. Zipfel, P.H., Modeling and Simulation of Aerospace Vehicle Dynamics, AIAA Educational Series, 2nd Ed., pp. 317-414 (2007). 
16. Ogata, K., Modern Control Engineering, Pearson Education, Prentice-Hall, 5th Ed., p. 171 (2009).

17. Colorni, A. Dorigo, M. and Maniezzo, V. "Distributed optimization by ant colonies", In Proceedings of the First European Conference on Artificial Life, Paris, France, pp. 134-142 (1991).

18. Glover, F. "Tabu search-part I", ORSA Journal on Computing, 1(3), pp. 190-206 (1989).

19. Pourtakdoust, S.H. and Nobahari, H. "An extension of ant colony system to continuous optimization problems", In Ant Colony Optimization and Swarm Intelligence, Brussels, Belgium, pp. 294-301 (2004).

\section{Appendix A}

\section{Derivation of translational equations}

To derive the translational equations of the flying vehicle, the two-body system is split at the bearing connection point and, then, the Newton's second law is written for each body. Eqs. (A.1) and (A.2) show the translational motion of front and aft bodies, respectively:

$$
\begin{aligned}
& m_{1} D^{E} \mathbf{v}_{B_{1}}^{E}=\mathbf{f}_{a_{1}}+m_{1} \mathbf{g}+\mathbf{f}_{12}, \\
& m_{2} D^{E} \mathbf{v}_{B_{2}}^{E}=\mathbf{f}_{a_{2}}+\mathbf{f}_{p}+m_{2} \mathbf{g}+\mathbf{f}_{21},
\end{aligned}
$$

where $D^{E}$ is the rotational time derivative with respect to the Earth frame; $B_{1}$ and $B_{2}$ represent the mass centers of the front and aft bodies, respectively; $\mathbf{v}_{B_{1}}^{E}$ and $\mathbf{v}_{B_{2}}^{E}$ are the velocity vectors of the front and aft bodies with respect to the Earth frame; $\mathbf{f}_{a_{1}}$ and $\mathbf{f}_{a_{2}}$ are the vectors of aerodynamic forces applied to the front and aft bodies; $\mathbf{g}$ is the gravity vector; $\mathbf{f}_{p}$ is the thrust force applied to the aft body; and $\mathbf{f}_{12}$ and $\mathbf{f}_{21}$ are the vector forces applied to the front body from the aft body and to the aft body from the front body, respectively. The translational motion of the entire flying vehicle can be obtained by adding both equations. Notice that $\mathbf{f}_{12}$ and $\mathbf{f}_{21}$ are internal forces and are canceled. Therefore:

$$
m_{2} D^{E} \mathbf{v}_{B_{2}}^{E}+m_{1} D^{E} \mathbf{v}_{B_{1}}^{E}=\mathbf{f}_{a_{1}}+\mathbf{f}_{a_{2}}+\mathbf{f}_{p}+m_{1} \mathbf{g}+m_{2} \mathbf{g} .
$$

The velocity vectors, $\mathbf{v}_{B_{1}}^{E}$ and $\mathbf{v}_{B_{2}}^{E}$, can be related to the total velocity vector, $\left(\mathbf{v}_{B}^{E}\right)$, in the following manner:

$$
\begin{aligned}
\mathbf{v}_{B_{1}}^{E} & =D^{E} \mathbf{s}_{B_{1} E}=D^{E} \mathbf{s}_{B_{1} B}+D^{E} \mathbf{s}_{B E} \\
& =D^{B_{1}} \mathbf{s}_{B_{1} B}+\boldsymbol{\Omega}^{B_{1} E} \mathbf{s}_{B_{1} B}+D^{E} \mathbf{s}_{B E} \\
& =\mathbf{v}_{B_{1}}^{B_{1}}+\boldsymbol{\Omega}^{B_{1} E} \mathbf{s}_{B_{1} B}+\mathbf{v}_{B}^{E}, \\
\mathbf{v}_{B_{2}}^{E} & =D^{E} \mathbf{s}_{B_{2} E}=D^{E} \mathbf{s}_{B_{2} B}+D^{E} \mathbf{s}_{B E} \\
& =D^{B_{1}} \mathbf{s}_{B_{2} B}+\boldsymbol{\Omega}^{B_{1} E} \mathbf{s}_{B_{2} B}+D^{E} \mathbf{s}_{B E} \\
& =\mathbf{v}_{B_{2}}^{B_{1}}+\boldsymbol{\Omega}^{B_{1} E} \mathbf{s}_{B_{2} B}+\mathbf{v}_{B}^{E},
\end{aligned}
$$

where $\mathbf{s}_{B_{1} E}$ and $\mathbf{s}_{B_{2} E}$ are the displacements of $B_{1}$ and $B_{2}$ with respect to an Earth-fixed point $(E) ; \mathbf{s}_{B_{1} B}$ and $\mathbf{s}_{B_{2} B}$ are the displacements of $B_{1}$ and $B_{2}$ with respect to the total mass center of the flying vehicle $(B) ; \mathbf{s}_{B E}$ is the displacement of $B$ with respect to $E ; D^{B_{1}}$ is the rotational time derivative with respect to the front body frame; $\Omega^{B_{1} E}$ is the skew symmetric form of the angular velocity vector of frame $B_{1}$ with respect to frame $E$; and $\mathbf{v}_{B_{1}}^{B_{1}}$ and $\mathbf{v}_{B_{2}}^{B_{1}}$ are the velocity vectors of $B_{1}$ and $B_{2}$ with respect to the front body frame. In this work, the velocity of mass centers is supposed to be negligible; thus, $\mathbf{v}_{B_{1}}^{B_{1}}$ and $\mathbf{v}_{B_{2}}^{B_{1}}$ will vanish. Now, substituting Eqs. (A.4) and (A.5) into Eq. (A.3) the following is obtained:

$$
\begin{gathered}
m_{2} D^{E}\left(\boldsymbol{\Omega}^{B_{1} E} \mathbf{s}_{B_{2} B}\right)+m D^{E} \mathbf{v}_{B}^{E}+m_{1} D^{E}\left(\boldsymbol{\Omega}^{B_{1} E} \mathbf{s}_{B_{1} B}\right) \\
=\mathbf{f}_{a_{1}}+\mathbf{f}_{a_{2}}+\mathbf{f}_{p}+m \mathbf{g} .
\end{gathered}
$$

The left hand of Eq. (A.6) is rewritten using Euler transformation as follows:

$$
\begin{aligned}
& m_{2} D^{B_{1}}\left(\boldsymbol{\Omega}^{B_{1} E} \mathbf{s}_{B_{2} B}\right)+m_{2} \boldsymbol{\Omega}^{B_{1} E} \boldsymbol{\Omega}^{B_{1} E} \mathbf{s}_{B_{2} B} \\
& +m_{1} D^{B_{1}}\left(\boldsymbol{\Omega}^{B_{1} E} \mathbf{s}_{B_{1} B}\right)+m_{1} \boldsymbol{\Omega}^{B_{1} E} \boldsymbol{\Omega}^{B_{1} E} \mathbf{s}_{B_{1} B} \\
& +m D^{B_{1}} \mathbf{v}_{B}^{E}+m \boldsymbol{\Omega}^{B_{1} E} \mathbf{v}_{B}^{E}=m \mathbf{g}+\mathbf{f}_{a_{1}}+\mathbf{f}_{a_{2}}+\mathbf{f}_{p} .
\end{aligned}
$$

The first and third terms of the left-hand side can be expanded in front body as follows:

$$
\begin{aligned}
& m_{2} D^{B_{1}}\left(\boldsymbol{\Omega}^{B_{1} E} \mathbf{s}_{B_{2} B}\right)+m_{1} D^{B_{1}}\left(\boldsymbol{\Omega}^{B_{1} E} \mathbf{s}_{B_{1} B}\right) \\
&=m_{2}\left(D^{B_{1}}\left(\boldsymbol{\Omega}^{B_{1} E}\right) \mathbf{s}_{B_{2} B}+\boldsymbol{\Omega}^{B_{1} E} D^{B_{1}} \mathbf{s}_{B_{2} B}\right) \\
& \quad+m_{1}\left(D^{B_{1}}\left(\boldsymbol{\Omega}^{B_{1} E}\right) \mathbf{s}_{B_{1} B}+\boldsymbol{\Omega}^{B_{1} E} D^{B_{1}} \mathbf{s}_{B_{1} B}\right) \\
&=m_{2} D^{B_{1}}\left(\boldsymbol{\Omega}^{B_{1} E}\right) \mathbf{s}_{B_{2} B}+m_{2} \boldsymbol{\Omega}^{B_{1} E} \mathbf{v}_{B_{2}}^{B_{1}} \\
&+m_{1} D^{B_{1}}\left(\boldsymbol{\Omega}^{B_{1} E}\right) \mathbf{s}_{B_{1} B}+m_{1} \boldsymbol{\Omega}^{B_{1} E} \mathbf{v}_{B_{2}}^{B_{1}} \\
&= m_{2} D^{B_{1}}\left(\boldsymbol{\Omega}^{B_{1} E}\right) \mathbf{s}_{B_{2} B}+m_{1} D^{B_{1}}\left(\boldsymbol{\Omega}^{B_{1} E}\right) \mathbf{s}_{B_{1} B} .
\end{aligned}
$$

The parameters of Eq. (A.8) can be expressed in front body coordinate as follows:

$$
\begin{aligned}
& {\left[\mathbf{\Omega}^{B_{1} E}\right]^{B_{1}}=\left[\begin{array}{ccc}
0 & -r & q \\
r & 0 & -p \\
-q & p & 0
\end{array}\right],} \\
& {\left[\mathbf{s}_{B_{1} B}\right]^{B_{1}}=\left[\begin{array}{c}
x_{1} \\
0 \\
0
\end{array}\right], \quad\left[\mathbf{s}_{B_{2} B}\right]^{B_{1}}=\left[\begin{array}{c}
-x_{2} \\
0 \\
0
\end{array}\right],}
\end{aligned}
$$

where $x_{1}=\frac{m_{2}}{m} d_{12}$, and $x_{2}=\frac{m_{1}}{m} d_{12}$. Substituting Eqs. (A.9) and (A.10) into Eq. (A.8), it can be shown 
that Eq. (A.8) will be zero. Similarly, it can be shown that the second and forth terms in the left-hand side of Eq. (A.7) will vanish. Finally, Eq. (A.7) is written as follows:

$$
m D^{B_{1}} \mathbf{v}_{B}^{E}+m \boldsymbol{\Omega}^{B_{1} E} \mathbf{v}_{B}^{E}=m \mathbf{g}+\mathbf{f}_{a_{1}}+\mathbf{f}_{a_{2}}+\mathbf{f}_{p} .
$$

This equation can be expressed in front body coordinate $]^{B_{1}}$ as follows:

$$
\begin{aligned}
{\left[\frac{d \mathbf{v}_{B}^{E}}{d t}\right]^{B_{1}} } & +\left[\mathbf{\Omega}^{B_{1} E}\right]^{B_{1}}\left[\mathbf{v}_{B}^{E}\right]^{B_{1}}=[T]^{B_{1} L}[\mathbf{g}]^{L} \\
& +\left(\frac{1}{m}\right)\left(\left[\mathbf{f}_{a_{1}}\right]^{B_{1}}+\left[\mathbf{f}_{a_{2}}\right]^{B_{1}}+\left[\mathbf{f}_{p}\right]^{B_{1}}\right)
\end{aligned}
$$

where $]^{L}$ represents the local-level coordinate and:

$$
\begin{aligned}
& {\left[\mathbf{v}_{B}^{E}\right]^{B_{1}}=\left[\begin{array}{c}
u \\
v \\
w
\end{array}\right]} \\
& {\left[\mathbf{f}_{a_{1}}\right]^{B_{1}}=\left[\begin{array}{c}
X_{1} \\
Y_{1} \\
Z_{1}
\end{array}\right]} \\
& {\left[\mathbf{f}_{a_{2}}\right]^{B_{1}}=[T]^{B_{1} B_{2}}\left[\mathbf{f}_{a_{2}}\right]^{B_{2}}=\left[\begin{array}{c}
X_{2} \\
Y_{2}^{\prime} \\
Z_{2}^{\prime}
\end{array}\right],} \\
& {\left[\mathbf{f}_{p}\right]^{B_{2}}=[T]^{B_{1} B_{2}}\left[\mathbf{f}_{p}\right]^{B_{2}}=\left[\begin{array}{c}
X_{T_{2}} \\
Y_{T_{2}}^{\prime} \\
Z_{T_{2}}^{\prime}
\end{array}\right] \text {, }} \\
& {[\mathbf{g}]^{L}=\left[\begin{array}{l}
0 \\
0 \\
g
\end{array}\right]} \\
& {[T]^{B_{1} L}=\left[\begin{array}{c}
\cos \psi \cos \theta \\
\cos \psi \sin \theta \sin \phi-\sin \psi \cos \phi \\
\cos \psi \sin \theta \cos \phi+\sin \psi \sin \phi
\end{array}\right.} \\
& \sin \psi \cos \theta \\
& \sin \psi \sin \theta \sin \phi+\cos \psi \cos \phi \\
& \sin \psi \sin \theta \cos \phi-\cos \psi \sin \phi \\
& \left.\begin{array}{c}
-\sin \theta \\
\cos \theta \sin \phi \\
\cos \theta \cos \phi
\end{array}\right] \\
& {[T]^{B_{1} B_{2}}=\left[\begin{array}{ccc}
1 & 0 & 0 \\
0 & \cos \phi_{r} & -\sin \phi_{r} \\
0 & \sin \phi_{r} & \cos \phi_{r}
\end{array}\right] \text {. }}
\end{aligned}
$$

By substituting Eq. (A.9), and Eqs. (A.13) to (A.17) into Eq. (A.12), Eqs. (1) to (3) are derived.

\section{Appendix B}

\section{Derivation of rotational equations}

Like the translational equations, to derive the rotational equations of the flying vehicle, the two-body system is split at the bearing connection point and, then, the Newton Euler's law is written for each body. The rotational motions of front and aft bodies can be stated as:

$$
\begin{aligned}
\mathbf{I}_{B_{1}}^{B_{1}} D^{B_{1}} \boldsymbol{\omega}^{B_{1} E} & +\boldsymbol{\Omega}^{B_{1} E} \mathbf{I}_{B_{1}}^{B_{1}} \boldsymbol{\omega}^{B_{1} E}=\mathbf{m}_{a_{1}}+\mathbf{m}_{12} \\
& +\mathbf{S}_{C B_{1}} \mathbf{f}_{12}, \\
\mathbf{I}_{B_{2}}^{B_{2}} D^{B_{2}} \boldsymbol{\omega}^{B_{2} E} & +\boldsymbol{\Omega}^{B_{2} E} \mathbf{I}_{B_{2}}^{B_{2}} \boldsymbol{\omega}^{B_{2} E}=\mathbf{m}_{a_{2}}+\mathbf{m}_{p} \\
& +\mathbf{m}_{21}+\mathbf{S}_{C B_{2}} \mathbf{f}_{21},
\end{aligned}
$$

where $\mathbf{I}_{B_{1}}^{B_{1}}$ and $\mathbf{I}_{B_{2}}^{B_{2}}$ are inertia tensors of $B_{1}$ and $B_{2}$ frames with respect to points $B_{1}$ and $B_{2}$, respectively, $\mathbf{m}_{a_{1}}$ and $\mathbf{m}_{a_{2}}$ are aerodynamic moments applied to front and aft bodies, $\mathbf{m}_{12}$ and $\mathbf{m}_{21}$ are the moments applied to the front body from the aft body and vice versa, $\mathbf{S}_{C B_{1}}$ and $\mathbf{S}_{C B_{2}}$ are the skew symmetric form of the displacement vectors from the front and aft mass centers to the bearing coupling point, $D^{B_{2}}$ is the rotational time derivative with respect to the aft body frame, $\boldsymbol{\omega}^{B_{2} E}$ and $\boldsymbol{\Omega}^{B_{2} E}$ are the vector and the skew symmetric forms of angular velocity vector of frame $B_{2}$ with respect to frame $E$, respectively, and $\mathbf{m}_{p}$ is the thrust moment applied to the aft body. These equations can be expressed in the front body coordinate ]$^{B_{1}}$ as follows:

$$
\begin{gathered}
{\left[\mathbf{I}_{B_{1}}^{B_{1}}\right]^{B_{1}}\left[\frac{d \boldsymbol{\omega}^{B_{1} E}}{d t}\right]^{B_{1}}+\left[\boldsymbol{\Omega}^{B_{1} E}\right]^{B_{1}}\left[\mathbf{I}_{B_{1}}^{B_{1}}\right]^{B_{1}}\left[\boldsymbol{\omega}^{B_{1} E}\right]^{B_{1}}} \\
=\left[\mathbf{m}_{a_{1}}\right]^{B_{1}}+\left[\mathbf{m}_{12}\right]^{B_{1}}+\left[\mathbf{S}_{C B_{1}}\right]^{B_{1}}\left[\mathbf{f}_{12}\right]^{B_{1}} \\
{\left[\mathbf{I}_{B_{2}}^{B_{2}}\right]^{B_{1}}[T]^{B_{1} B_{2}}\left[\frac{d \boldsymbol{\omega}^{B_{2} E}}{d t}\right]^{B_{2}}} \\
+\left[\mathbf{\Omega}^{B_{2} E}\right]^{B_{1}}\left[\mathbf{I}_{B_{2}}^{B_{2}}\right]^{B_{1}}\left[\boldsymbol{\omega}^{B_{2} E}\right]^{B_{1}}=\left[\mathbf{m}_{a_{2}}\right]^{B_{1}} \\
+\left[\mathbf{m}_{p}\right]^{B_{1}}+\left[\mathbf{m}_{21}\right]^{B_{1}}+\left[\mathbf{S}_{C B_{2}}\right]^{B_{1}}\left[\mathbf{f}_{21}\right]^{B_{1}}
\end{gathered}
$$

Given the assumptions made in Section 2, the parameters of Eqs. (B.3) and (B.4) can be expressed in $]^{B_{1}}$ as follows:

$$
\left[\mathbf{m}_{a_{1}}\right]^{B_{1}}=\left[\begin{array}{c}
L_{1} \\
M_{1} \\
N_{1}
\end{array}\right]
$$




$$
\begin{aligned}
& {\left[\mathbf{m}_{a_{2}}\right]^{B_{1}}=[T]^{B_{1} B_{2}}\left[\mathbf{m}_{a_{2}}\right]^{B_{2}}=\left[\begin{array}{c}
L_{2} \\
M_{2}^{\prime} \\
N_{2}^{\prime}
\end{array}\right],} \\
& {\left[\mathbf{m}_{p}\right]^{B_{1}}=[T]^{B_{1} B_{2}}\left[\mathbf{m}_{p}\right]^{B_{2}}=\left[\begin{array}{c}
L_{T_{2}} \\
M_{T_{2}}^{\prime} \\
N_{T_{2}}^{\prime}
\end{array}\right],} \\
& {\left[\mathbf{f}_{12}\right]^{B_{1}}=\left[\begin{array}{l}
f_{12_{x}} \\
f_{12_{y}} \\
f_{12_{z}}
\end{array}\right], \quad\left[\mathbf{f}_{21}\right]^{B_{1}}=\left[\begin{array}{l}
f_{21_{x}} \\
f_{21_{y}} \\
f_{21_{z}}
\end{array}\right],} \\
& {\left[\mathbf{m}_{12}\right]^{B_{1}}=-\left[\mathbf{m}_{21}\right]^{B_{1}}=\left[\begin{array}{c}
b_{t} \dot{\phi}_{r} \\
M_{12} \\
N_{12}
\end{array}\right],} \\
& {\left[\mathbf{I}_{B_{1}}^{B_{1}}\right]^{B_{1}}=\left[\begin{array}{ccc}
I_{x_{1}} & 0 & 0 \\
0 & I_{y_{1}} & 0 \\
0 & 0 & I_{y_{1}}
\end{array}\right],} \\
& {\left[\mathbf{I}_{B_{2}}^{B_{2}}\right]^{B_{1}}=[T]^{B_{1} B_{2}}\left[\mathbf{I}_{B_{2}}^{B_{2}}\right]^{B_{2}}[T]^{B_{2} B_{1}}} \\
& =\left[\begin{array}{ccc}
I_{x_{2}} & 0 & 0 \\
0 & I_{y_{2}} & 0 \\
0 & 0 & I_{y_{2}}
\end{array}\right] \text {, } \\
& {\left[\mathbf{S}_{C B_{1}}\right]^{B_{1}}=\left[\begin{array}{ccc}
0 & 0 & 0 \\
0 & 0 & d_{1} \\
0 & -d_{1} & 0
\end{array}\right] \text {, }} \\
& {\left[\mathbf{S}_{C B_{2}}\right]^{B_{1}}=\left[\begin{array}{ccc}
0 & 0 & 0 \\
0 & 0 & -d_{2} \\
0 & d_{2} & 0
\end{array}\right],}
\end{aligned}
$$

where $b_{t}$ is the viscous friction coefficient of the bearing, and $d_{1}$ and $d_{2}$ are distances between the mass centers of the front and aft bodies and midpoint of bearing, respectively. To calculate $\left[\boldsymbol{\omega}^{B_{2} E}\right]^{B_{1}}, \boldsymbol{\omega}^{B_{2} E}$ is expanded as:

$$
\boldsymbol{\omega}^{B_{2} E}=\boldsymbol{\omega}^{B_{2} B_{1}}+\boldsymbol{\omega}^{B_{1} E}
$$

This equation can be expressed in the front body coordinates $]^{B_{1}}$ as follows:

$$
\left[\boldsymbol{\omega}^{B_{2} E}\right]^{B_{1}}=\left[\boldsymbol{\omega}^{B_{2} B_{1}}\right]^{B_{1}}+\left[\boldsymbol{\omega}^{B_{1} E}\right]^{B_{1}}
$$

where:

$$
\begin{aligned}
& {\left[\boldsymbol{\omega}^{B_{2} E}\right]^{B_{1}}=[T]^{B_{1} B_{2}}\left[\boldsymbol{\omega}^{B_{2} E}\right]^{B_{2}}=\left[\begin{array}{c}
p_{2} \\
q_{2}^{\prime} \\
r_{2}^{\prime}
\end{array}\right],} \\
& {\left[\boldsymbol{\omega}^{B_{2} B_{1}}\right]^{B_{1}}=\left[\begin{array}{c}
\dot{\phi}_{r} \\
0 \\
0
\end{array}\right] .}
\end{aligned}
$$

Therefore:

$$
\left[\boldsymbol{\omega}^{B_{2} E}\right]^{B_{1}}=\left[\begin{array}{c}
p_{2} \\
q_{2}^{\prime} \\
r_{2}^{\prime}
\end{array}\right]=\left[\begin{array}{c}
p+\dot{\phi}_{r} \\
q \\
r
\end{array}\right]=\left[\begin{array}{c}
p_{2} \\
q \\
r
\end{array}\right] .
$$

In a similar way, $[T]^{B_{1} B_{2}}\left[\frac{d \omega^{B_{2} E}}{d t}\right]^{B_{2}}$ can be calculated as follows:

$$
\begin{gathered}
D^{B_{2}} \boldsymbol{\omega}^{B_{2} E}=D^{B_{1}} \boldsymbol{\omega}^{B_{2} E}+\boldsymbol{\Omega}^{B_{1} B_{2}} \boldsymbol{\omega}^{B_{2} E}, \\
{[T]^{B_{1} B_{2}}\left[\frac{d \boldsymbol{\omega}^{B_{2} E}}{d t}\right]^{B_{2}}=\left[\frac{d \boldsymbol{\omega}^{B_{2} E}}{d t}\right]^{B_{1}}} \\
+\left[\boldsymbol{\Omega}^{B_{1} B_{2}}\right]^{B_{1}}\left[\boldsymbol{\omega}^{B_{2} E}\right]^{B_{1}}
\end{gathered}
$$

or:

$$
[T]^{B_{1} B_{2}}\left[\frac{d \boldsymbol{\omega}^{B_{2} E}}{d t}\right]^{B_{2}}=\left[\begin{array}{c}
\dot{p}_{2} \\
\dot{q}+\dot{\phi}_{r} r \\
\dot{r}-\dot{\phi}_{r} q
\end{array}\right] .
$$

By substituting Eqs. (B.5) to (B.11), (B.16), and (B.19) into Eqs. (B.3) and (B.4), the component form of these equations can be written as:

$$
\begin{aligned}
& {\left[\begin{array}{ccc}
I_{x_{1}} & 0 & 0 \\
0 & I_{y_{1}} & 0 \\
0 & 0 & I_{y_{1}}
\end{array}\right]\left[\begin{array}{c}
\dot{p} \\
\dot{q} \\
\dot{r}
\end{array}\right]} \\
& +\left[\begin{array}{ccc}
0 & -r & q \\
r & 0 & -p \\
-q & p & 0
\end{array}\right]\left[\begin{array}{ccc}
I_{x_{1}} & 0 & 0 \\
0 & I_{y_{1}} & 0 \\
0 & 0 & I_{y_{1}}
\end{array}\right]\left[\begin{array}{l}
p \\
q \\
r
\end{array}\right] \\
& =\left[\begin{array}{c}
L_{1} \\
M_{1} \\
N_{1}
\end{array}\right]+\left[\begin{array}{c}
b_{t} \dot{\phi}_{r} \\
M_{12} \\
N_{12}
\end{array}\right]+\left[\begin{array}{ccc}
0 & 0 & 0 \\
0 & 0 & d_{1} \\
0 & -d_{1} & 0
\end{array}\right]\left[\begin{array}{l}
f_{12_{x}} \\
f_{12_{y}} \\
f_{12_{z}}
\end{array}\right], \\
& {\left[\begin{array}{ccc}
I_{x_{2}} & 0 & 0 \\
0 & I_{y_{2}} & 0 \\
0 & 0 & I_{y_{2}}
\end{array}\right]\left[\begin{array}{c}
\dot{p}_{2} \\
\dot{q}+\dot{\phi}_{r} r \\
\dot{r}-\dot{\phi}_{r} q
\end{array}\right]} \\
& +\left[\begin{array}{ccc}
0 & -r & q \\
r & 0 & -p_{2} \\
-q & p_{2} & 0
\end{array}\right]\left[\begin{array}{ccc}
I_{x_{2}} & 0 & 0 \\
0 & I_{y_{2}} & 0 \\
0 & 0 & I_{y_{2}}
\end{array}\right]\left[\begin{array}{c}
p_{2} \\
q \\
r
\end{array}\right] \\
& =\left[\begin{array}{c}
L_{2} \\
M_{2}^{\prime} \\
N_{2}^{\prime}
\end{array}\right]+\left[\begin{array}{c}
L_{T_{2}} \\
M_{T_{2}}^{\prime} \\
N_{T_{2}}^{\prime}
\end{array}\right]+\left[\begin{array}{l}
-b_{t} \dot{\phi}_{r} \\
-M_{12} \\
-N_{12}
\end{array}\right] \\
& +\left[\begin{array}{ccc}
0 & 0 & 0 \\
0 & 0 & -d_{2} \\
0 & d_{2} & 0
\end{array}\right]\left[\begin{array}{l}
f_{21_{x}} \\
f_{21_{y}} \\
f_{21_{z}}
\end{array}\right] .
\end{aligned}
$$

Eqs. (4) and (5) can be concluded from Eqs. (B.20) and (B.21). Moreover:

$$
\begin{aligned}
& I_{y_{1}}(\dot{q}-p r)+I_{x_{1}} p r=M_{1}+d_{1} f_{12_{z}}, \\
& I_{y_{2}}(\dot{q}-p r)+I_{x_{2}} p_{2} r=M_{2}^{\prime}+M_{T_{2}}^{\prime}-d_{2} f_{21_{z}} .
\end{aligned}
$$


Adding these equations provides Eq. (6) as follows:

$$
\begin{array}{r}
I_{y_{1}}(\dot{q}-p r)+I_{x_{1}} p r+I_{y_{2}}(\dot{q}-p r)+I_{x_{2}} p_{2} r \\
=M_{1}+M_{2}^{\prime}+M_{T_{2}}^{\prime}-d_{12} f_{21_{z}} .
\end{array}
$$

By substituting Eqs. (A5) and (1) to (3) into Eq. (A.2) and performing a series of mathematical operations, the last term of Eq. (B.24) can be expressed as follows:

$$
\begin{aligned}
-d_{12} f_{21_{z}}= & -x_{1} Z_{1}+x_{2} Z^{\prime}+x_{2} Z_{T_{2}} \\
& -\left(\frac{m_{1} m_{2}}{m}\right) d_{12}^{2}(\dot{q}-p r) .
\end{aligned}
$$

By substituting Eq. (B.25) into Eq. (B.24) and performing a series of mathematical operations, Eq. (6) is derived. Eq. (7) can be achieved in a similar way.

\section{Biographies}

Hadi Nobahari is an Assistant Professor in the Department of Aerospace Engineering at Sharif University of Technology. He received his BS, MS, and PhD degrees in Aerospace Engineering, Flight Dynamic, and Control Division from Sharif University of Technology in 1998, 2000, and 2006, respectively. He is now the Director of Guidance and Control Research Center. His research interests include novel applications of heuristic algorithms in aerospace, intelligent guidance and control systems, and cooperative guidance and navigation systems.

Mojtaba Arab Kermani received his MSc degrees in Aerospace Engineering from Sharif University of Technology, Tehran, Iran, in 2014. His main research areas are flight dynamics, modeling and simulation of aerospace vehicles, and optimal control. 